\title{
TU/e EmonONEN

\section{A variational approach to magnetoelastic buckling problems for systems of superconducting tori}

\section{Citation for published version (APA):}

Smits, P. R. J. M., Lieshout, van, P. H., \& Ven, van de, A. A. F. (1988). A variational approach to magnetoelastic buckling problems for systems of superconducting tori. (RANA : reports on applied and numerical analysis; Vol. 8809). Technische Universiteit Eindhoven.

\section{Document status and date:}

Published: 01/01/1988

\section{Document Version:}

Publisher's PDF, also known as Version of Record (includes final page, issue and volume numbers)

\section{Please check the document version of this publication:}

- A submitted manuscript is the version of the article upon submission and before peer-review. There can be important differences between the submitted version and the official published version of record. People interested in the research are advised to contact the author for the final version of the publication, or visit the $\mathrm{DOI}$ to the publisher's website.

- The final author version and the galley proof are versions of the publication after peer review.

- The final published version features the final layout of the paper including the volume, issue and page numbers.

Link to publication

\section{General rights}

Copyright and moral rights for the publications made accessible in the public portal are retained by the authors and/or other copyright owners and it is a condition of accessing publications that users recognise and abide by the legal requirements associated with these rights.

- Users may download and print one copy of any publication from the public portal for the purpose of private study or research.

- You may not further distribute the material or use it for any profit-making activity or commercial gain

- You may freely distribute the URL identifying the publication in the public portal.

If the publication is distributed under the terms of Article $25 \mathrm{fa}$ of the Dutch Copyright Act, indicated by the "Taverne" license above, please follow below link for the End User Agreement:

www.tue.nl/taverne

Take down policy

If you believe that this document breaches copyright please contact us at:

openaccess@tue.nl

providing details and we will investigate your claim. 


\author{
RANA 88-09 \\ May 1988 \\ A variational approach \\ to magnetoelastic buckling problems \\ for systems of superconducting tori \\ by \\ P.R.J.M. Smits \\ P.H. van Lieshout \\ A.A.F. van de Ven
}

Reports on Applied and Numerical Analysis

Department of Mathematics and Computing Science

Eindhoven University of Technology

P.O. Box 513

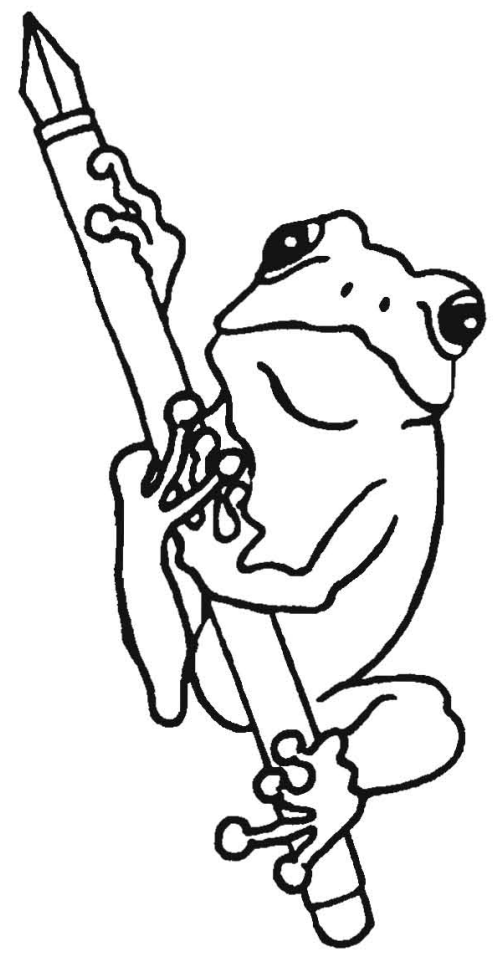

$5600 \mathrm{MB}$ Eindhoven

The Netherlands 


\section{Introduction}

In two recent papers, [1], [2], by Van Lieshout, Rongen and Van de Ven, the problem of magnetoelastic buckling was studied on the basis of a variational aproach. In [1] a variational principle, yielding explicit relations for magnetoelastic buckling values, was formulated and in [2] applications to systems of ferromagnetic or superconducting beams were presented. As one of the results of [2], it was proved that a configuration of two equal parallel superconducting rods could become unstable, and the pertinent buckling value for the current was calculated. The mechanical stability of supercuuducting structures has been subject of an increasing amount of research; an excellent survey of this field is given by F. Moon in his monograph [3]. As one of the many subjects in [3], the stability of toroidal superconductors in a transverse or a toroidal magnetic field was discussed. The stability of one superconducting torus in its own field was investigated by Chattopadhyay, [4], and by Van de Ven and Couwenberg, [5], both leading to the conclusion that the natural configuration of the torus was stable.

In this paper we apply a Legendre transformation to the variational principle in [1], and we thus obtain a second variational principle. This principle is believed to be more suitable for numerical purposes, because it contains constraints on the fundamental variables which are much weaker than the constraints in [1]. As in [1], [2] and [5] we assume that the electric current is confined to the surface of the superconducting body. In order to arrive at analytical expressions for buckling values, we set up two integral equations, one for the surface current density $\mathbf{J}$ and one for a variable $\tilde{\psi}$, which is related to the perturbation potential $\psi$.

We shall examine two specific buckling problems for superconducting systems. The first concerns in-plane buckling of a pair of concentric tori, and the second out-of-plane buckling of a coaxial pair of equal tori. All tori have equal circular cross-sections. In both cases a small parameter $\varepsilon$ is introduced representing the slenderness of the system. In the integral equations for $\mathbf{J}$ and $\tilde{\psi}$ the integration over the tangential coordinate $\phi$ is carried out exactly, and then the integral equations are linearized with respect to $\varepsilon$. It appears that in both cases $\boldsymbol{J}$ and $\tilde{\psi}$, and therefore also the magnetic field in initial and perturbed state, are in zeroeth order in $\varepsilon$ the same as in the case of two parallel rods (see [2]). When the currents in the two tori are equally directed (which is the technically relevant case) only these zeroeth order fields together with the elastic energy of the buckling mode, play a role in the computation of the buckling value. Therefore, the buckling values of two concentric and two coaxial tori differ only a numerical factor from the buckling value of an equivalent pair of parallel rods. The numerical factor depends on the elastic energy only. When the currents in the two tori are directed opposite to each other, higher order developments of $\mathbf{J}$ and $\tilde{\psi}$ are needed, and the analysis becomes laborious. For these cases, we confine ourselves to stating that if the tori will buckle at all, the buckling value is much higher than in the case of equally directed currents. In conclusion, we present some numerical results and we compare these results with those obtained from a mathematically less complicated, but also less rigorous, method. This method, which was also applied in [2], is based upon a generalization of the law of Biot and Savart (cf. [3], (2-6.4)). In general, the correspondence between the results of the two methods is good. 


\section{A variational principle}

Consider a superconducting body, on the surface of which a current flows with density $\mathbf{J}$ per unit of length. The deformed configuration of the body is denoted by $G^{-}$, its boundary by $\partial G$ and the vacuum outside the body by $G^{+}$. In [1] a variational principle has been derived, that could serve as the basis for a magnetoelastic stability theory for a superconducting body. The Lagrangian densities $\mathrm{L}^{+}$and $\mathrm{L}^{-}$outside and inside the body, respectively, are given by (see [1], (7.2))

$$
\mathrm{L}^{+}=-\frac{1}{2 \mu_{0}}(\mathbf{B}, \mathbf{B}), \quad \mathbf{L}^{-}=-\rho U
$$

accompanied by the constraints ([1], (7.3)-(7.4))

$$
\begin{array}{lll}
\operatorname{div} \mathbf{B}=0, & \mathbf{x} \in G^{+} ; \quad \mathbf{B}=0, \quad T=\rho \frac{\mathrm{d} U}{\mathrm{~d} F} F^{T}, \quad \rho J_{F}=\rho_{0}, \quad \mathbf{x \in G ^ { - }} ; \\
(\mathbf{B}, \mathrm{n})=0, \quad \mathbf{x} \in \partial G ; & \mathbf{B} \rightarrow 0, \quad|\mathbf{x}| \rightarrow \infty .
\end{array}
$$

In (2.1)-(2.2), $B$ is the magnetic induction, $T$ the Cauchy stress tensor, $\rho$ and $\rho_{0}$ the mass densities in the deformed and undeformed state, respectively, $U$ the internal energy density, $F$ the deformation gradient, $J_{F}=\operatorname{det} F$ the Jacobian and $\mathbf{n}$ the outward unit normal on $\partial G$. Here, the upper indices ${ }^{+}$and ${ }^{-}$on $\mathbf{B}$ are omitted.

The variational principle based on (2.1)-(2.2), as described in [1], can be used to solve the buckling problem of the superconductor. But then, as already mentioned in the Note following [1], (7.16), there is no freedom left for variation of the magnetic potential, which is awkward in numerical applications. This difficulty can be smoothed over using a Legendre transformation, or transformation into the reciprocal form (see [6], Ch. IV, §9). The formal procedure for Legendre transformation is as follows. Firstly, we pass from the variable $\mathbf{B}$ to the variable $\mathbf{H}$ defined by (see [6], Ch. IV, (87))

$$
\mathbf{H}=\frac{1}{\mu_{0}} \mathbf{B}\left[=\frac{\mathrm{d}}{\mathrm{d} \mathbf{B}}\left[\frac{1}{2 \mu_{0}}(\mathbf{B}, \mathbf{B})\right]\right] \text {. }
$$

Of course, $\mathbf{H}$ is the magnetic field intensity. Secondly, we add a term $(\mathbf{H}, \mathbf{B})$ to the Lagrangian density $\mathrm{L}^{+}$and, thirdly, we replace the constraints $\operatorname{div} B=0$ and $(B, n)=0$ by the constraint curl $\mathbf{H}=0$. As in our case $\mathbf{B}$ and $\mathbf{H}$ only differ a fixed multiplicative constant $\mu_{0}$, we can hold on to $\mathbf{B}$ as our fundamental variable. The Legendre transformation then amounts to a change of sign in the outer Lagrangian density,

$$
\mathbf{L}^{+}=\frac{1}{2 \mu_{0}}(\mathbf{B}, \mathbf{B}), \quad \mathbf{L}^{-}=-\rho U,
$$

while the constraints become

$$
\begin{aligned}
& \mathbf{B}=0, \quad T=\rho \frac{\mathrm{d} U}{\mathrm{~d} F} F^{T}, \quad \rho J_{F}=\rho_{0}, \quad \mathbf{x} \in G^{-} ; \\
& \operatorname{curl} \mathbf{B}=0, \quad \mathbf{x} \in G^{+} ; \quad \mathbf{B} \rightarrow 0, \quad|\mathbf{x}| \rightarrow \infty .
\end{aligned}
$$

As an extra constraint we prescribe the total current $I_{0}$ by means of Ampere's law, i.e. 


$$
\int_{C}(\mathbf{B}, \tau) d s=\mu_{0} I_{0}
$$

where $C$ is a contour entirely in the vacuum and $\tau$ is the tangent vector at $C$. The contour $C$ has to be suitably chosen for the specific problem at hand.

In the same way as done in [1], we can formulate on the basis of (2.4)-(2.6) a variational principle that can be used in the study of the buckling problem for a superconducting body. Note that the inner Lagrangian density $\mathrm{L}^{-}$in (2.4) is equal to the one in (2.1), which at his turn is equal to $\mathrm{L}^{-}$according to [1], (3.1), provided that in the latter the internal field $\mathrm{H}^{-}$and the field at infinity $B_{0}$ are taken equal to zero. Hence, we can adopt the calculation of the difference $L^{-}-L^{\sigma}$ of the inner Lagrangians in [1], Section 3. Putting equal to zero all internal magnetic field quantities and $B_{0}$ we obtain from [1], (3.27) (with the notations according to [1], and with omission of the upperindices 9

$$
L^{-}-L^{\sigma}=\delta L^{-}+J^{-}+O\left(\varepsilon^{3}\right)
$$

where

$$
\delta L^{-}=\int_{G^{-}} T_{i j j} u_{i} d V-\int_{\mathcal{L}} T_{i j} N_{j} u_{i} d S
$$

and

$$
J^{-}=-\frac{1}{2} \int_{G^{-}} \rho c_{i j k} u_{i, k} u_{j, l} d V .
$$

Since the outer Lagrangian densities in (2.1) and (2.4) only differ in their sign, we can use the calculation of $L^{+}-L^{0^{+}}$in [1]. Taking $B_{0}=0, \mathrm{H}=\mathrm{B} / \mu_{0}$, and $e_{i j k} a_{k j}^{+}=b_{i}$ in [1], (3.39), and multiplying its right-hand side by -1 , we obtain

$$
\begin{aligned}
& L^{+}-L^{0^{+}}=\frac{1}{\mu_{0}} \int_{G^{+}} b_{i} B_{i} d V-\frac{1}{2 \mu_{0}} \int_{\alpha G} B_{k} B_{k} u_{i} N_{i} d S \\
& -\frac{1}{\mu_{0}} \int_{\alpha G}\left[b_{k} B_{k} u_{i}+\frac{1}{2} B_{k, j} B_{k} u_{i} u_{j}+\frac{1}{4} B_{k} B_{k}\left(u_{i} u_{j j}-u_{j} u_{i, j}\right)\right] N_{i} d S \\
& +\frac{1}{2 \mu_{0}} \int_{G^{+}} b_{i} b_{i} d V .
\end{aligned}
$$

Since the constraints (2.5)-(2.6) have to be satisfied for both the intermediate and the present state, the constraints for the perturbations are

$$
\begin{aligned}
& \mathbf{b}=0, \quad t_{i j}=-T_{i j} u_{k, k}+T_{i k} u_{j, k}+\rho c_{i k j l} u_{k \downarrow}, \quad \xi \in G^{-} \\
& \operatorname{curl} \mathbf{b}=0, \quad \mathbf{x} \in G^{+} ; \quad \mathbf{b} \rightarrow 0, \quad|x| \rightarrow \infty,
\end{aligned}
$$

and

$$
\int^{(b, \tau) d s=0 .}
$$

The constraints $(2.9)^{3}$ and (2.10) guarantee the existence of a continuous potential $\psi(\mathbf{x}), \mathbf{x} \in G^{+}$, such that 


$$
\mathbf{b}=\nabla \psi, \quad \mathbf{x} \in G^{+} .
$$

To dispose of irrelevant constants in $\psi$, we replace $(2.9)^{4}$ by the constraint

$$
\psi \rightarrow 0, \quad|x| \rightarrow \infty .
$$

Addition of (2.7.1) and (2.8), after the use of (2.11), yields

$$
L-L^{0}=\delta L+J+O\left(\varepsilon^{3}\right)
$$

where

$$
\delta L=\int_{G^{-}} T_{i j, j} u_{i} d V-\int_{\mathcal{L}}\left(T_{i j} N_{j}+\frac{1}{2 \mu_{0}} B_{k} B_{k} N_{i}\right) u_{i} d S+\frac{1}{\mu_{0}} \int_{G^{+}} \Psi_{i} B_{i} d V,
$$

and

$$
\begin{aligned}
& J=-\frac{1}{2} \int_{G^{-}} \rho c_{i j d t} u_{i, k} u_{j, l} d V \\
& -\frac{1}{\mu_{0}} \int_{G G}\left[\Psi, k B_{k} u_{i}+\frac{1}{2} B_{k, j} B_{k} u_{i} u_{j}+\frac{1}{4} B_{k} B_{k}\left(u_{i} u_{j, j}-u_{j} u_{i, j}\right)\right] N_{i} d S \\
& +\frac{1}{2 \mu_{0}} \int_{G^{+}} \Psi_{, i} \Psi_{, i} d V .
\end{aligned}
$$

The Legendre transformation ensures us that our variational principle is equivalent to the variational principle in [1]. This means that variation of $L$ and $J$ results in sets of equations fully describing the magnetoelastic buckling of the superconducting body. Of course, it is also possible to verify this directly. Using Gauss's divergence theorem in the last term of (2.13.2), we can show that the variation $\delta L$ of $L$ is equal to

$$
\begin{aligned}
\delta L= & \int_{G^{-}} T_{i j j} u_{i} d V-\int_{d G}\left[\left(T_{i j} N_{j}+\frac{1}{2 \mu_{0}} B_{k} B_{k} N_{i}\right) u_{i}+\frac{1}{\mu_{0}} \psi B_{i} N_{i}\right] d S \\
& -\frac{1}{\mu_{0}} \int_{G^{+}} \psi B_{i, i} d V .
\end{aligned}
$$

Variation of $L$, i.e. the requirement $\delta L=0$ for all $\mathbf{u}$ and $\psi$, yields the remaining intermediate equations

$$
\begin{aligned}
& T_{i j, j}=0, \quad \xi \in G^{-} ; \\
& B_{i} N_{i}=0, \quad T_{i j} N_{j}+\frac{1}{2 \mu_{0}} B_{k} B_{k} N_{i}=0, \quad \xi \in \partial G ; \\
& B_{i, i}=0, \quad x \in G^{+} .
\end{aligned}
$$

With use of the same instruments as in [1], e.g. Gauss's divergence theorem and the lemma following [1], (3.35), it is possible to show that variation of $J$ yields the remaining perturbed equations (compare with the results of [1], Section 4) 


$$
\begin{aligned}
& t_{i j, j}-T_{i k, j} u_{j, k}=0, \quad \xi \in G^{-} \\
& \left(t_{i j}-t_{i j}^{M}\right) N_{j}-\left(T_{i k}-T_{i k}^{M}\right) u_{j, k} N_{j}-T_{i j, k}^{M} u_{k} N_{j}=0, \\
& \Psi_{, i} N_{i}+B_{i, j} u_{j} N_{i}-B_{j} u_{i, j} N_{i}=0, \quad \xi \in \partial G ; \\
& \Psi_{, i i}=0, \quad x \in G^{+} .
\end{aligned}
$$

In order to obtain a suitable form of the buckling equation $J=0$, we rewrite expression (2.13.3). We assume that the superconducting body is isotropic, homogeneous and linearly elastic. As in [1], we neglect intermediate deformations, and thus identify the intermediate configuration and the undeformed or natural configuration of the body. Then (cf. [1], (6.16))

$$
\rho c_{i j k l} u_{i, k} u_{j, l}=T_{j k} u_{i, j} u_{i, k}+\frac{E}{1+v}\left[\frac{v}{1-2 v} e_{k k} e_{l l}+e_{k l} e_{k l}\right)
$$

where $E$ is Young's modulus, $v$ is Poisson's ratio and

$$
e_{i j}:=\frac{1}{2}\left(u_{i, j}+u_{j, i}\right) \text {. }
$$

Furthermore, we rewrite half of the second term in the right-hand side of (2.13.3) as follows (with the aid of the lemma following [1], (3.35), and $\left.(2.15)^{2,4}\right)$,

$$
\begin{aligned}
& -\frac{1}{2 \mu_{0}} \int_{\partial G} \Psi_{,} B_{k} u_{i} N_{i} d S=-\frac{1}{2 \mu_{0} \partial G}\left\{\left(\psi B_{j} u_{i}\right)_{j}-\psi B_{j, j} u_{i}-\psi B_{j} u_{i, j}\right\} N_{i} d S \\
& =\frac{1}{2 \mu_{0}} \int_{\partial G}\left(B_{i, j} u_{j}-B_{j} u_{i, j}\right) \psi N_{i} d S .
\end{aligned}
$$

Substituting (2.17) and (2.19) into (2.13.3) we obtain

$$
\begin{aligned}
& J=-\frac{1}{2} \int_{G^{-}}\left[T_{j k} u_{i, j} u_{i, k}+\frac{E}{1+v}\left[\frac{v}{1-2 v} e_{k k} e_{l l}+e_{k l} e_{k l}\right]\right] d V \\
& -\frac{1}{2 \mu_{0}} \int_{\delta G}\left[\psi_{j} B_{j} u_{i}+\left(B_{i, j} u_{j}-B_{j} u_{i, j}\right) \psi+B_{k j} B_{k} u_{i} u_{j}+\frac{1}{2} B_{k} B_{k}\left(u_{i} u_{j, j}-u_{j} u_{i, j}\right)+\psi \psi_{, i}\right] N_{i} d S \\
& -\frac{1}{2 \mu_{0}} \int_{G^{+}} \Psi \Psi_{, i i} d V .
\end{aligned}
$$

For the first term of the first integral we use Gauss's divergence theorem together with $(2.15)^{1,3}$. On account of curl $B=0$ we have

$$
B_{i, j}=B_{j, i}
$$

which we apply to the third term in the second integral in (2.20). Rearranging terms, we finally arrive at the identity

$$
\begin{aligned}
& J=\frac{1}{2} \int_{G^{-}}\left[T_{j k} u_{i j k} u_{i} \frac{E}{1+v}\left[\frac{v}{1-2 v} e_{k k} e_{l l}+e_{k l} e_{k l}\right)\right] d V \\
& \frac{1}{2 \mu_{0}} \int_{\delta G}\left[\left(\psi+B_{k} u_{k}\right)_{j} B_{j} u_{i}+\frac{1}{2} B_{k} B_{k}\left(u_{j, j} u_{i}-u_{i, j} u_{j}-u_{j, i} u_{j}\right)\right.
\end{aligned}
$$




$$
\left.-\frac{1}{2} B_{j} B_{k}\left(u_{j, k}+u_{k, j}\right) u_{i}+\left(\psi_{, i}+B_{i, j} u_{j}-B_{j} u_{i, j}\right) \psi\right] N_{i} d S-\frac{1}{2 \mu_{0}} \int_{G^{+}} \psi \Psi_{, i i} d V
$$

In order to dispose of the integral over the infinite region $G^{+}$, we impose $(2.16)^{4}$ as an extra constraint on $\psi$, so that the constraints for $\psi$ now are

$$
\Delta \psi=0, \quad \mathbf{x} \in G^{+} ; \quad \psi \rightarrow 0, \quad|\mathbf{x}| \rightarrow \infty .
$$

In contrast to [1], (7.15), $\psi$ is not completely determined by (2.23), so that there is still freedom for variation. In analogy with [2], (3.1), we introduce the normalized variables

$$
\hat{\mathbf{B}}:=\frac{2 \pi a}{\mu_{0} I_{0}} \mathbf{B}, \quad \hat{T}:=\frac{(2 \pi a)^{2}}{\mu_{0} I_{0}^{2}} T, \quad \hat{\psi}:=\frac{2 \pi a}{\mu_{0} I_{0}} \psi,
$$

where $a$ is some length-parameter, which has to be chosen suitably for the problem under consideration. Thus the buckling equation $J=0$ yields (immediately omitting the hats)

$$
\begin{aligned}
& \frac{E(2 \pi a)^{2}}{\mu_{0} J_{0}^{2}}=\left\{\int _ { \partial G } \left[-\left(\Psi+B_{k} u_{k}\right)_{j} B_{j} u_{i}-\frac{1}{2} B_{k} B_{k}\left(u_{j, j} u_{i}-u_{j} u_{i, j}-u_{j} u_{j, i}\right)\right.\right. \\
& \left.\left.+\frac{1}{2} B_{j} B_{k}\left(u_{j, k}+u_{k, j}\right) u_{i}-\left(\Psi_{, i}+B_{i j} u_{j}-B_{j} u_{i j}\right) \Psi\right] N_{i} d S+\int_{G^{-}} T_{j k} u_{i, j k} u_{i} d V\right\} \\
& \left\{\frac{1}{1+v} \int_{G^{-}}\left[\frac{v}{1-2 v} e_{k k} e_{l l}+e_{k l} e_{k l}\right] d V\right\}^{-1} .
\end{aligned}
$$

We have already noted that this formula for the buckling value $I_{0}$ is especially useful when it is difficult to determine $\psi$ exactly. If, however, we are able to calculate $\psi$ exactly, as we shall do in the next sections, then the last term in the first integral in (2.25) drops out, and formula (2.25) becomes equivalent to [1], (7.18). It is in this latter form that we shall use the above relation in the next sections. 


\section{Integral equations}

Since $(B, N)=0$ on $\partial G$, the derivative of $\psi+(B, u)$ occurring in the first term in the right-hand side of (2.25) reduces to a purely tangential derivative. Hence, knowledge of the values of $\mathbf{B}$ and $\psi+(\mathbf{B}, \mathbf{u})$ on $\partial G$ suffices for the calculation of the right-hand side of (2.25) (with the last term in the first integral equal to zero). These values can be calculated from two integral equations, derived below, namely one for the surface current density $J$, which is related to the magnetic induction $B$ on $\partial G$ by (cf. [1], (7.1))

$$
\mu_{0} \mathrm{~J}=\mathbf{N} \times \mathbf{B}, \quad \text { or } \mathbf{B}=\mu_{0} \mathrm{~J} \times \mathbf{N}, \quad \mathbf{x} \in \partial G,
$$

and one for the variable $\tilde{\psi}$, defined on $\partial G$ by

$$
\tilde{\psi}:=\psi+(\mathbf{B}, \mathbf{u}), \quad \mathrm{x} \in \partial G,
$$

which we call the modified perturbation potential. From [7], Sec. 4.12, form. (3), we see that

$$
\mathbf{B}\left(\mathbf{x}_{0}\right)=\mu_{0} \int_{\mathcal{L}} \mathrm{J}(\mathbf{x}) \times \nabla_{x} G\left(\mathbf{x}, \mathbf{x}_{0}\right) d S_{x}, \quad \mathbf{x}_{0} \in G^{+},
$$

where

$$
G\left(\mathbf{x}, \mathbf{x}_{0}\right)=\frac{1}{4 \pi\left|\mathbf{x}-\mathbf{x}_{0}\right|}, \quad \mathbf{x} \neq \mathbf{x}_{0},
$$

is the fundamental solution of Laplace's equation. Using the theory of single layer and double layer potentials (see e.g. [8], Chap. 11), we can determine the limiting behaviour of the integral in (3.3) when $\mathrm{x}_{0}$ tends to a point on $\partial G$. Taking the limit $\mathrm{x}_{0} \rightarrow \partial G$ in (3.3) and using (3.1) we arrive at the first integral equation

$$
\mathrm{J}\left(\mathbf{x}_{0}\right) \times \mathbf{N}\left(\mathbf{x}_{0}\right)=2 \int_{\partial G} \mathrm{~J}(\mathbf{x}) \times \nabla_{x} G\left(\mathbf{x}, \mathbf{x}_{0}\right) d S_{x}, \quad \mathbf{x}_{0} \in \partial G
$$

On account of the equation $\Delta \psi=0$ in $G^{+}$and the condition $\psi \rightarrow 0$ for $|\mathbf{x}| \rightarrow \infty$, Green's second identity implies (compare with [2], (3.33)-(3.34))

$$
\psi\left(\mathbf{x}_{0}\right)=\int_{\partial G}\left[\psi(\mathbf{x}) \frac{\partial G}{\partial N_{x}}\left(\mathbf{x}, \mathbf{x}_{0}\right)-\frac{\partial \psi}{\partial N_{x}}(\mathbf{x}) G\left(\mathbf{x}, \mathbf{x}_{0}\right)\right] d S_{x}, \quad \mathbf{x}_{0} \in G^{+} .
$$

Analogously to (3.5) we find by letting $x_{0}$ tend towards a point on $\partial G$ the integral representation for $\psi$,

$$
\Psi\left(\mathbf{x}_{0}\right)=2 \int_{\partial G}\left[\Psi(\mathbf{x}) \frac{\partial G}{\partial N_{x}}\left(\mathbf{x}, \mathbf{x}_{0}\right) \frac{\partial \psi}{\partial N_{x}}(\mathbf{x}) G\left(\mathbf{x}, \mathbf{x}_{0}\right)\right] d S_{x}, \quad \mathbf{x}_{0} \in \partial G .
$$

Using subsequently $(2.16)^{3},(2.15)^{2,4}$ and (3.1) we can derive

$$
\begin{aligned}
& -\int_{\alpha G} \frac{\partial \psi}{\partial N_{x}}(\mathrm{x}) G\left(\mathrm{x}, \mathrm{x}_{0}\right) d S_{x}=\int_{\mathcal{L}}\left(B_{i, j} u_{j}-B_{j} u_{i, j}\right) G N_{i} d S_{x} \\
& =\int_{\mathcal{L}} B_{j} u_{i} G_{j} N_{i} d S_{x}
\end{aligned}
$$




$$
=\int_{\delta G}\left[-\mu_{0}\left(\mathbf{u}(\mathbf{x}) \times \mathbf{J}(\mathbf{x}), \nabla_{x} G\left(\mathbf{x}, \mathbf{x}_{0}\right)\right)+(\mathbf{B}(\mathbf{x}), \mathbf{u}(\mathbf{x})) \frac{\partial G}{\partial N_{x}}\left(\mathbf{x}, \mathbf{x}_{0}\right)\right] d S_{x} .
$$

Substitution of (3.8) into (3.7) yields

$$
\begin{aligned}
\psi\left(\mathbf{x}_{0}\right)= & 2 \int_{\partial G}\left[(\psi(\mathbf{x})+(\mathbf{B}(\mathbf{x}), \mathbf{u}(\mathbf{x}))) \frac{\partial G}{\partial N_{x}}\left(\mathbf{x}, \mathbf{x}_{0}\right)\right. \\
& \left.-\mu_{0}\left(\mathbf{u}(\mathbf{x}) \times \mathbf{J}(\mathbf{x}), \nabla_{x} G\left(\mathbf{x}, \mathbf{x}_{0}\right)\right)\right] d S_{x}, \quad \mathbf{x}_{0} \in \partial G .
\end{aligned}
$$

Taking the inner product with $\mu_{0} u\left(x_{0}\right)$ on toth sides of (3.5), using (3.1), and adding the resulting equation to (3.9), we finally arrive at the second integral equation

$$
\begin{aligned}
\tilde{\psi}\left(\mathbf{x}_{0}\right)=2 \int_{\partial G}\left[\tilde{\psi}(\mathbf{x}) \frac{\partial G}{\partial N_{x}}\left(\mathbf{x}, \mathbf{x}_{0}\right)\right. \\
\left.+\mu_{0}\left(\left(\mathbf{u}\left(\mathbf{x}_{0}\right)-\mathbf{u}(\mathbf{x})\right) \times \mathbf{J}(\mathbf{x}), \nabla_{x} G\left(\mathbf{x}, \mathbf{x}_{0}\right)\right)\right] d S_{x}, \quad \mathbf{x}_{0} \in \partial G .
\end{aligned}
$$

In the next two sections we shall use (3.5) and (3.10) to determine the exact $\mathbf{J}$ and $\tilde{\psi}$ for sets of two concentric and two coaxial tori, respectively. 


\section{Two concentric superconducting tori}

Consider two concentric superconducting tori, which both have a circular cross-section with radius $a$. The central line of the outer torus has radius $b+c$, and the central line of the inner torus has radius $b-c$, where $c>a$. A coordinate system $\left\{O \mathrm{e}_{r} \mathrm{e}_{\phi} \mathrm{e}_{2}\right\}$ is chosen with $O$ in the joint centre of the two central lines, $e_{r}$ and $e_{\phi}$ in the equatorial plane (i.e. the plane through the central lines) and $e_{z}$ perpendicular to the equatorial plane. The corresponding cylindrical coordinates are denoted by $(r, \phi, z)$. A cross-section of the pair of tori is shown in Fig.1.

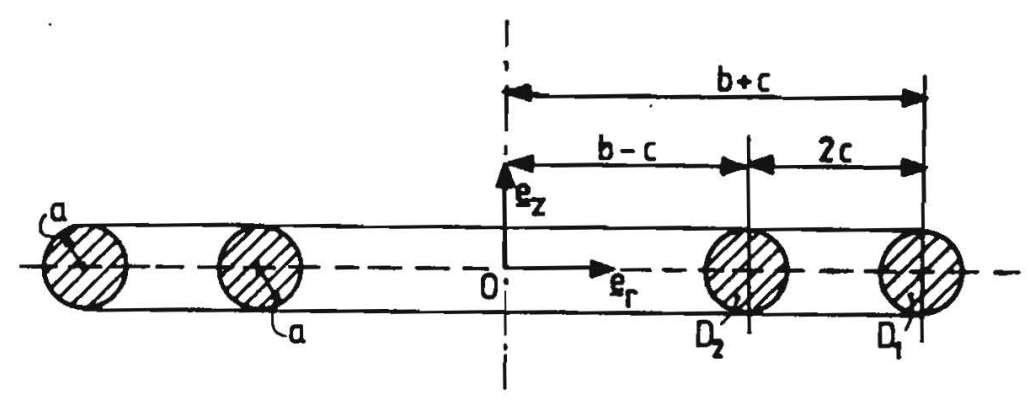

Fig.1. Cross-section of a pair of concentric tori.

The interiors of the outer and inner torus are denoted by $G_{1}^{-}$and $G_{2}^{-}$and their boundaries by $\partial G_{1}$ and $\partial G_{2}$, respectively. The intersections of the outer and inner torus with the half-plane $\phi=0$ are denoted by $D_{1}^{-}$and $D_{2}^{-}$, with boundaries $\partial D_{1}$ and $\partial D_{2}$, respectively. We define $D^{-}:=D_{1}^{-} \cup D_{2}^{-}$and $\partial D:=\partial D_{1} \cup \partial D_{2}$. We suppose that

$$
\varepsilon:=\frac{a}{b} \ll 1,
$$

and furthermore that

$$
m:=\frac{c}{a}=O(1), \quad(m>1) .
$$

In view of (4.1)-(4.2), the system of the two tori is called slender, and $\varepsilon$ is called the slenderness-parameter.

In the intermediate state a current flows on the surfaces of the two tori with surface current density

$$
J=J(r, z) e_{\phi} .
$$

The total current on the outer torus has the prescribed value $I_{0}$. The total current on the inner torus is taken equal to $I_{0}$ or $-I_{0}$; the currents on the two tori are called equally directed or oppositely directed, respectively. Because of rotational symmetry in the intermediate state, the Cauchy stresses $T_{r \phi}$ and $T_{\phi z}$ and the magnetic field component $B_{\phi}$ vanish and all intermediate fields are independent of $\phi$.

We only consider in-plane buckling. The deflection of the central line of the outer $(i=1)$ and inner torus $(i=2)$ can then be written as $w_{i}(\phi) e_{r}+v_{i}(\phi) e_{\phi}$. In analogy with Bernoulli's theory for the bending of slender inextensible beams, the displacement fields of the tori (considered as slender rings) may be written as (neglecting $O\left(\varepsilon^{3}\right)$-terms) 


$$
\begin{array}{ll}
u_{r}=w_{i}+\frac{v}{2} \frac{\left(r-b_{i}\right)^{2}-z^{2}}{b_{i}^{2}}\left(w^{\prime \prime}{ }_{i}-v_{i}^{\prime}\right), & \\
u_{\phi}=v_{i}-\frac{r-b_{i}}{b_{i}}\left(w_{i}^{\prime}-v_{i}\right), & *) \\
u_{z}=v \frac{z\left(r-b_{i}\right)}{b_{i}^{2}}\left(w^{\prime \prime}{ }_{i}-v_{i}^{\prime}\right), & \text { in } G_{i}^{-}, i=1,2,
\end{array}
$$

where

$$
b_{1}=b+c, b_{2}=b-c,
$$

while the inextensibility of the rings is expressed by the condition

$$
v_{i}^{\prime}(\phi)+w_{i}(\phi)=0, \quad i=1,2 .
$$

The perturbation potential $\psi(r, \phi, z)$ is separated according to

$$
\psi(r, \phi, z)=\zeta(r, z) \omega(\phi) \text {. }
$$

Using $\Delta \psi=0$ and the periodicity of $\omega(\phi)$, i.e. $\omega(\phi+2 \pi)=\omega(\phi)$, we find that $\omega(\phi)$ must be of the form

$$
\omega(\phi)=\Omega \cos (n \phi+\alpha),
$$

where $n$ is a natural number. We take $\alpha=0$; this is always possible by redefining the coordinate $\phi$. Substitution of (4.4) and (4.8) into the boundary condition $(2.16)^{3}$ for $\partial \psi / \partial N$, reveals that this boundary condition can only be satisfied for every $\phi \in[0,2 \pi]$ if $w_{1}(\phi)$ and $w_{2}(\phi)$ are proportional to $\cos n \phi$. In the sequel we take $n=2$, which corresponds to the first bending mode. Thus,

$$
w_{i}(\phi)=w_{i} \cos 2 \phi, \quad i=1,2 .
$$

Because of $B_{\phi}=0$, the separation (4.7) induces a separation of $\tilde{\psi}=\psi+(B, u)$, which we write as follows

$$
\tilde{\psi}(r, \phi, z)=f(r, z) W \cos 2 \phi, \quad W:=\sqrt{W_{1}^{2}+W_{2}^{2}} .
$$

The ratio $W_{2} / W_{1}$ is yet unknown. Since (2.25) is based upon a variational principle, we can determine this ratio by variation of the right-hand side of $(2.25)$.

We identify the parameter $a$ in (2.24)-(2.25) with the radius $a$ of the cross-sections of the tori. Furthermore, in addition to (2.24) we introduce the dimensionless variables (which will be used continually in the sequel without explicit use of the hats)

$$
\hat{\mathrm{J}}:=\frac{2 \pi a}{I_{0}} \mathrm{~J}, \quad \hat{J}(r, z):=\frac{2 \pi a}{I_{0}} J(r, z), \hat{f}(r, z):=\frac{2 \pi a}{\mu_{0} J_{0}} f(r, z) .
$$

We proceed with the determination of zeroeth order approximations with respect to $\varepsilon$ for $J$ and $f$. It turns out that these approximations for $J$ and $f$ are identical to the corresponding functions for the case of two slender parallel beams, as calculated in [2].

*) In the sequel we do not apply the summation convention with respect to the indices $i$ and $j$. 


\subsection{The zeroeth order approximation of $J(r, z)$}

The current density $\mathbf{J}$ can be determined from (3.5). Since $J\left(x_{0}\right)$ is independent of $\phi_{0}$, we may confine ourselves to $\phi_{0}=0$. Putting

$$
x=r e_{r}+z e_{z}, \quad x_{0}=r_{0} e_{r_{0}}+z_{0} e_{z_{0}},
$$

where, for $\phi_{0}=0$,

$$
\mathbf{e}_{\tau_{0}}=\cos \phi e_{r}-\sin \phi e_{\phi}, \quad \mathbf{e}_{\phi_{0}}=\sin \phi e_{r}+\cos \phi e_{\phi}, \quad \mathbf{e}_{z_{0}}=\mathbf{e}_{2},
$$

we obtain from (3.4)

$$
\begin{aligned}
& \nabla_{x} G\left(\mathrm{x}, \mathrm{x}_{0}\right)=\frac{\mathrm{x}_{0}-\mathrm{x}}{4 \pi\left|\mathrm{x}_{0}-\mathrm{x}\right|^{3}} \\
& =\frac{1}{4 \pi R^{3}}\left(\left(r_{0} \cos \phi-r\right) \mathrm{e}_{r}-r_{0} \sin \phi \mathrm{e}_{\phi}+\left(z_{\sigma}-z\right) \mathrm{e}_{z}\right),
\end{aligned}
$$

with

$$
R:=\left|\mathbf{x}_{\sigma} \mathbf{x}\right|=\sqrt{r_{0}^{2}+r^{2}-2 r_{0} r \cos \phi+\left(z_{0}-z\right)^{2}} .
$$

For the normal vector $\mathbf{N}\left(\mathrm{x}_{0}\right)$ in (3.5) we substitute

$$
\mathbf{N}\left(\mathbf{x}_{0}\right)=N_{r_{0}} \mathbf{e}_{r_{0}}+N_{z_{0}} \mathbf{e}_{z_{0}},
$$

while for the surface element $d S_{x}$ we have

$$
d S_{x}=r d \phi d s,
$$

in which $d s$ is the line-element on the boundary $\partial D$ of the cross-section $D^{-}$. Taking the inner product with $e_{\phi_{0}} \times \mathbf{N}\left(\mathbf{x}_{0}\right)$ on both sides of (3.5), and using (4.3) and (4.12)-(4.17), we derive

$$
J\left(r_{0}, z_{0}\right)=\frac{1}{2 \pi} \int_{\partial D} \int_{0}^{2 \pi} \frac{N_{r_{0}}\left(r_{0} \cos \phi-r\right)+N_{z_{0}}\left(z_{\sigma}-z\right) \cos \phi}{R^{3}} J(r, z) r d \phi d s .
$$

The integration over $\phi$ can be carried out exactly. For an arbitrary integrable function $g(\phi)$

$$
\int_{0}^{2 \pi} \frac{g(\phi)}{R^{3}} d \phi=\frac{k^{3}}{4(r \sigma)^{3 / 2}} \int_{-1 / 2 \pi}^{1 / 2 \pi} \frac{g(\pi-2 \theta)}{\Delta^{3}} d \theta
$$

where

$$
\Delta:=\sqrt{1-k^{2} \sin ^{2} \theta}, \quad k:=\left[\frac{4 r_{0} r}{\left(r_{0}+r\right)^{2}+\left(z_{0}-z\right)^{2}}\right]^{1 / 2} .
$$

By means of (4.19), the integrals over $\phi$ occurring in (4.18) can be reduced to complete elliptic integrals of the first and second kind (cf. [9], pp. 904-905, for the relevant definitions). Formulae 37 and 42 from [9], 2.584, reveal that

$$
\int_{0}^{1 / 2 \pi} \frac{1}{\Delta^{3}} d \theta=\frac{1}{k^{2}} E(k),
$$




$$
\int_{0}^{1 / 2 \pi} \frac{\cos ^{2} \theta}{\Delta^{3}} d \theta=\frac{1}{k^{2}}(K(k)-E(k)),
$$

in which $K$ and $E$ are the complete elliptic integrals of the first and second kind, respectively, and

$$
k^{\prime}:=\sqrt{1-k^{2}}
$$

is the complementary modulus. Further, we define

$$
\alpha_{1}(k):=\int_{0}^{1 / 2 \pi} \frac{-2 \cos ^{2} \theta}{\Delta^{3}} d \theta=-\frac{2}{k^{2}}(K(k)-E(k)) .
$$

Substituting (4.19-24) into (4.18), rearranging terms and using the identity

$$
k^{2}=\frac{\left(r_{\sigma}-r\right)^{2}+\left(z_{0}-z\right)^{2}}{\left(r_{0}+r\right)^{2}+\left(z_{0}-z\right)^{2}}=\frac{k^{2}}{4 r_{\sigma^{r}}}\left(\left(r_{0}-r\right)^{2}+\left(z_{0}-z\right)^{2}\right) \text {, }
$$

we find the integral equation (omit the arguments of $J, E$ and $K$ )

$$
\begin{aligned}
J= & \frac{1}{2 \pi} \int_{\partial D} \frac{k^{3} r}{2\left(r_{0} r\right)^{3 / 2}}\left[\frac{4 r_{0} r}{k^{2}} \frac{N_{r_{0}}\left(r_{0}-r\right)+N_{z_{0}}\left(z_{0}-z\right)}{\left(r_{0}-r\right)^{2}+\left(z_{0}-z\right)^{2}} E\right. \\
& \left.+N_{r_{0}} r \alpha_{1}+\left(N_{r_{0}}\left(r_{0}-r\right)+N_{z_{0}}\left(z_{\sigma}-z\right)\right) \alpha_{1}\right] J d s .
\end{aligned}
$$

Before linearizing the above integrand with respect to $\varepsilon$, we introduce the notations

$$
\begin{aligned}
& r=b+a \xi, \quad z=a \eta, \quad r_{0}=b+a \xi_{0}, \quad z_{0}=a \eta_{0}, \\
& N_{\xi}=N_{r}, \quad N_{\eta}=N_{z}, \quad N_{\xi_{0}}=N_{r_{0}}, \quad N_{\eta_{0}}=N_{z_{0}}, \\
& d s=a d \lambda, \\
& h\left(\xi, \eta ; \xi_{0}, \eta_{0}\right)=\sqrt{\left(\xi_{0}-\xi\right)^{2}+\left(\eta_{0}-\eta\right)^{2}}, \\
& l_{0}\left(\xi, \eta ; \xi_{0}, \eta_{0}\right)=\frac{N_{\xi_{0}}\left(\xi_{0}-\xi\right)+N_{\eta_{0}}\left(\eta_{0}-\eta\right)}{\left(\xi_{0}-\xi\right)^{2}+\left(\eta_{0}-\eta\right)^{2}} .
\end{aligned}
$$

The contour defined by the points $(\xi, \eta)$ for which $(r, z) \in \partial D$, is called $C$, so $C=C_{1} \cup C_{2}$, where $C_{1}$ and $C_{2}$ are the circles

$$
C_{1}:(\xi-m)^{2}+\eta^{2}=1, \quad C_{2}:(\xi+m)^{2}+\eta^{2}=1 .
$$

In view of (4.2) both $\xi$ and $\eta$ are of order unity with respect to the small parameter $\varepsilon$ when $(\xi, \eta) \in C$. Then it it is easy to verify that $(4.25)$ implies

$$
k^{2}=\frac{1}{4} \varepsilon^{2} h^{2}\left(\xi, \eta ; \xi_{0}, \eta_{0}\right)(1+O(\varepsilon))
$$

so we can use the developments of $K$ and $E$ for small $k^{\prime}$ (see [9], 8.113, form. 3, and 8.114, form. 3), 


$$
K(k)=\ln \frac{4}{k^{\prime}}+O\left(k^{2} \ln k^{\prime}\right), \quad E(k)=1+O\left(k^{2} \ln k^{\prime}\right), \quad K \rightarrow 0 .
$$

From (4.33)-(4.34) we derive

$$
\begin{aligned}
& K(k)=\ln \frac{8}{\varepsilon}-\ln h+O(\varepsilon), \quad E(k)=1+O\left(\varepsilon^{2} \ln \varepsilon\right), \\
& k=1+O\left(\varepsilon^{2}\right), \quad \alpha_{1}(k)=-2 \ln \frac{8}{\varepsilon}+2 \ln h-2+O(\varepsilon) .
\end{aligned}
$$

The zeroeth order approximation with respect to $\varepsilon$ of $J$ is denoted by $J^{(0)}$, so

$$
J=J^{(0)}(1+O(\varepsilon)),
$$

Substitution of (4.27)-(4.31) into (4.26) and linearization with respect to $\varepsilon$ with the aid of (4.33)-(4.37), yields the simplified integral equation

$$
J^{(0)}=\frac{1}{\pi} \int_{C} l_{0} J^{(0)} d \lambda
$$

We introduce the complex variables

$$
\begin{aligned}
& z=\xi+i \eta, \quad z_{0}=\xi_{0}+i \eta_{0}, \\
& N=N_{\xi}+i N_{\eta}, \quad N_{0}=N_{\xi_{0}}+i N_{\eta_{0}}, \quad S=i N, \quad S_{0}=i N_{0},
\end{aligned}
$$

so that according to (4.30)-(4.31)

$$
h=\left|z_{0}-z\right|, \quad l_{0}=\operatorname{Re} \frac{N_{0}\left(\overline{z_{0}}-\bar{z}\right)}{\left|z_{0}-z\right|^{2}}=-\operatorname{Re} \frac{N_{0}}{z-z_{0}},
$$

where $\bar{z}$ and $\bar{z}_{0}$ denote the complex conjugates of $z$ and $z_{0}$. The contours $C_{1}$ and $C_{2}$ are given by

$$
C_{1}:|2-m|=1, C_{2}:|2+m|=1,
$$

while for $z \in C=C_{1} \cup C_{2}$

$$
d z=S d \lambda=i N d \lambda, \quad d \lambda=\bar{S} d z=-i \bar{N} d z .
$$

The exterior of $C$ is denoted by $S^{+}$and the interior of $C$ by $S^{-}$. Substitution of (4.41) and (4.43) into (4.38) transforms this integral equation into

$$
J^{(0)}\left(z_{0}\right)=\operatorname{Re}\left\{-\frac{N_{0}}{\pi i} f_{C} \frac{J^{(0)}(z)}{z-z_{0}} \bar{N} d z\right\}, \quad z_{0} \in C,
$$

where $f$ stands for Cauchy's principal value. To solve this integral equation, we introduce the Cauchy integral

$$
F\left(z_{0}\right)=\frac{1}{2 \pi} \int_{C} \frac{J^{(0)}(z)}{z-z_{0}} \bar{N} d z, \quad z_{0} \in C V C .
$$

The function $F(z)$ has the following properties (see [2], (3.55)-(3.57))

$$
F(z) \text { analytical, } \quad z \in S^{-} \cup S^{+} \text {, }
$$




$$
\begin{aligned}
& F(z)=O\left(z^{-1}\right), \quad z \rightarrow \infty, \\
& F^{-}\left(z_{0}\right)-F^{+}\left(z_{0}\right)=\dot{J}^{(0)}\left(z_{0}\right) \vec{N}_{0}, \quad z_{0} \in C, \\
& F^{-}\left(z_{0}\right)+F^{+}\left(z_{0}\right)=\frac{1}{\pi} \int_{C} \frac{J^{(0)}(z)}{z-z_{0}} \bar{N} d z, \quad z_{0} \in C,
\end{aligned}
$$

where $F^{-}$and $F^{+}$are defined by

$$
F^{ \pm}\left(z_{0}\right)=\lim _{z \rightarrow z_{0}, z \in S^{ \pm}} F(z), \quad z_{0} \in C .
$$

Because of (4.44) and (4.48)-(4.49) we have

$$
\begin{aligned}
& \operatorname{Re}\left(i F^{-} N\right\}=0, \text { on } C, \\
& \operatorname{Im}\left\{i F^{-} N-i F^{+} N\right\}=0, \text { on } C .
\end{aligned}
$$

The relations (4.46) (for $z \in S\urcorner$ ) and (4.51) constitute an interior Riemann-Hilbert problem (see [10], Chap. 5, §39) for $S^{-}$, with trivial solution

$$
F(z)=0, \quad z \in S^{-} \text {. }
$$

The relations (4.46) (for $z \in S^{+}$), (4.47) and (4.52) (in which we use (4.53) and (4.43)) constitute the following exterior Riemann-Hilbert problem:
i) $F(z)$ analytical, $z \in S^{+}$,
ii) $\operatorname{Im}\left\{F^{+} d z\right\}=0$, on $C$,
iii) $F(z)=O\left(z^{-1}\right), \quad z \rightarrow \infty$.

As extra constraint there still remains (2.6), saying that the total current over $\partial D_{1}$ must equal $I_{0}$, which under the neglect of $O(\varepsilon)$-terms yields (recall that here $J^{(0)}$ is the dimensionless current density according to (4.11))

$$
I_{0}=\frac{I_{0}}{2 \pi} \int_{C_{1}} J^{(0)} d \lambda=\frac{I_{0}}{2 \pi} \int_{C_{1}} F^{+}(z) d z,
$$

in which we have used (4.43), (4.48) and (4.53). Hence,

$$
\int_{1} F^{+}(z) d z=2 \pi
$$

and in the same way it follows that

$$
\int_{C_{2}} F^{+}(z) d z= \begin{cases}+2 \pi, & \left(\mathbf{S}_{\varrho}\right), \\ -2 \pi, & \left(\mathbf{S}_{\varrho}\right)\end{cases}
$$

where $\left(\mathbf{S}_{e}\right)$ indicates the case of equally directed currents and $\left(\mathbf{S}_{\varrho}\right)$ the case of opposite currents. Using the theory of [10], Chap. $5, \S 40$ and $\$ 42$, it can be shown that $F(z)$ is completely determined by the relations (4.54) and (4.56)-(4.57). We note that (4.54) and (4.56) are identical to the relations (3.26) (S) and (4.4) (S) of [2] and, furthermore, that (4.56)-(4.57) are in accordance with the symmetry relations [2], (4.3) $\left(\mathbf{S}_{e}, S_{0}\right)$, reading

$$
F(-z)=-F(z),\left(\mathrm{S}_{e}\right) ; \quad F(-z)=F(z), \quad\left(\mathrm{S}_{0}\right) .
$$

Hence, the present $F(z)$ and the one in [2], referring to the (S)-case, are necessarily the same. 
This fact essentially means that the zeroeth order approximations of the surface current density for two slender tori and for two slender beams are identical.

\subsection{The zeroeth order approximation of $f(r, z)$}

We proceed with the determination of the function $f(r, z)$ from (3.10), under the neglect of $O\left(\varepsilon^{2}\right)$-terms. For this we may again confine ourselves to $\phi_{0}=0$. In that case we deduce with (4.3), (4.4), (4.9) and (4.13)

$$
\begin{aligned}
\left(\mathbf{u}\left(\mathbf{x}_{0}\right)-\mathbf{u}(\mathbf{x})\right) \times \mathrm{J}(\mathbf{x})=\left(W_{i} \cos \phi-W_{j} \cos 2 \phi\right) J(r, z) \mathbf{e}_{2}, \\
\mathbf{x}_{0} \in \partial G_{i}, \mathbf{x} \in \partial G_{j}, i, j=1,2 .
\end{aligned}
$$

Substitution of (4.10), (4.14)-(4.17) and (4.59) into (3.10) for $\phi_{0}=0$ yields the integral equation for $f$ on $\partial D_{i}$

$$
\begin{aligned}
& W f\left(r_{0}, z_{0}\right)=\sum_{j=1}^{2} \frac{1}{2 \pi} \int_{\partial D_{j}} \int_{0}^{2 \pi}\left\{W f(r, z) \cos 2 \phi \frac{N_{r}\left(r_{0} \cos \phi-r\right)+N_{z}\left(z_{0}-z\right)}{R^{3}}\right. \\
& \left.+\left(W_{i} \cos \phi-W_{j} \cos 2 \phi\right) J(r, z) \frac{z_{0}-z}{R^{3}}\right\} r d \phi d s, \quad\left(r_{0}, z_{0}\right) \in \partial D_{i} .
\end{aligned}
$$

Again we carry out the integration over $\phi$ exactly. To this end we first calculate with the aid of (4.19) and (4.21) the integrals

$$
\int_{0}^{2 \pi} \frac{\cos 2 \phi}{R^{3}} d \phi=\frac{k^{3}}{2\left(r_{0}\right)^{3 / 2}}\left[\frac{1}{k^{2}} E(k)+\alpha_{2}(k)\right],
$$

and

$$
\int_{0}^{2 \pi} \frac{\cos 2 \phi \cos \phi}{R^{3}} d \phi=\frac{k^{3}}{2\left(r_{\sigma} r\right)^{3 / 2}}\left[\frac{1}{k^{2}} E(k)+\alpha_{3}(k)\right]
$$

where

$$
\alpha_{2}(k)=\int_{0}^{1 / 2 \pi} \frac{8 \cos ^{4} \theta-8 \cos ^{2} \theta}{\Delta^{3}} d \theta
$$

and

$$
\alpha_{3}(k)=\int_{0}^{1 / 2 \pi} \frac{-16 \cos ^{6} \theta+24 \cos ^{4} \theta-10 \cos ^{2} \theta}{\Delta^{3}} d \theta .
$$

The functions $\alpha_{2}(k)$ and $\alpha_{3}(k)$ can be expressed in terms of $E(k)$ and $K(k)$, but we only need the asymptotic behaviour of $\alpha_{2}(k)$ and $\alpha_{3}(k)$ for $k \rightarrow 1$. The asymptotic behaviour of the integrals of $1 / \Delta^{3}$ and $\cos ^{2} \theta / \Delta^{3}$ can be deduced directly from (4.21)-(4.22) and (4.34), and the asymptotic behaviour of the integrais of $\cos ^{4} \theta / \Delta^{3}$ and $\cos ^{6} \theta / \Delta^{3}$ can be computed elementarily, noting that $\cos ^{4} \theta / \Delta^{3}$ and $\cos ^{6} \theta / \Delta^{3}$ are bounded for $0 \leq \theta \leq 1 / 2 \pi, 0 \leq k \leq 1$. Therefore, we only give the results here, reading 


$$
\begin{aligned}
& \alpha_{2}(k)=-8 \ln \frac{4}{k^{\prime}}+16+O\left(k^{2} \ln k^{\prime}\right), \\
& \alpha_{3}(k)=-10 \ln \frac{4}{k^{\prime}}+\frac{70}{3}+O\left(k^{2} \ln k^{\prime}\right) .
\end{aligned}
$$

Substitution of (4.21), (4.24) and (4.61)-(4.62) into (4.60) yields

$$
\begin{aligned}
& W f=\sum_{j=1}^{2} \int_{\partial b_{j}} \frac{k^{3} r}{2\left(r_{\sigma}\right)^{3 / 2}}\left\{W f \left[\frac{4 r_{0} r}{k^{2}} \frac{N_{r}\left(r_{0}-r\right)+N_{z}\left(z_{0}-z\right)}{\left(r_{\sigma}-r\right)^{2}+\left(z_{0}-z\right)^{2}} E\right.\right. \\
& \left.+N_{r} r_{0}\left(\alpha_{3}-\alpha_{2}\right)+\left(N_{r}\left(r_{\sigma}-r\right)+N_{z}\left(z_{\sigma}-z\right)\right) \alpha_{2}\right] \\
& \left.+J\left(z_{\sigma}-z\right)\left[\frac{W_{i}-W_{j}}{k^{2}} E+W_{i} \alpha_{1}-W_{j} \alpha_{2}\right]\right\} d s, \quad \text { on } \partial D_{i}, i=1,2
\end{aligned}
$$

In addition to (4.27)-(4.31) we introduce

$$
l\left(\xi, \eta ; \xi_{0}, \eta_{0}\right)=\frac{N_{\xi}\left(\xi_{0}-\xi\right)+N_{\eta}\left(\eta_{0}-\eta\right)}{\left(\xi_{0}-\xi\right)^{2}+\left(\eta_{0}-\eta\right)^{2}},
$$

and we denote the zeroeth order approximation of the function $f(r, z)$ by $f^{(0)}(r, z)$, so

$$
f(r, z)=f^{(0)}(r, z)(1+O(\varepsilon)) \text {. }
$$

Developing the integrand of (4.67) for small $\varepsilon$, with the aid of (4.35)-(4.37) and (4.65)-(4.66), we find the following integral equation for $f^{(0)}$

$$
\begin{aligned}
& f^{(0)}=\sum_{j=1}^{2} \frac{1}{\pi} \int_{C_{j}}\left\{l f^{(0)}+\frac{1}{4} J^{(0)}\left(\eta_{\sigma}-\eta\right) \varepsilon^{2}\left[\frac{W_{i}-W_{j}}{W} \frac{4}{\varepsilon^{2} h^{2}}\right.\right. \\
& \left.\left.+\frac{W_{i}}{W}\left(-2 \ln \frac{8}{\varepsilon h}+2\right)-\frac{W_{j}}{W}\left(-8 \ln \frac{8}{\varepsilon h}+16\right)\right]\right\} d \lambda, \text { on } C_{i}, i=1,2 .
\end{aligned}
$$

If $W_{1}=W_{2}$, i.e. if the two tori have the same buckling patterns, the $O\left(\varepsilon^{-2}\right)$-term between [ ] vanishes for all $i$ and $j$, and then $f^{(0)}$ will be $O\left(\varepsilon^{2}\right)$ smaller than in the case $W_{1} \neq W_{2}$. In the next subsection it turns out that the order of magnitude of the lowest buckling value is directly related to the order of $f^{(0)}$ and, therefore, we are primarily interested in the lowest order terms of $f^{(0)}$. This brings us to assume

$$
q:=\frac{W_{1}-W_{2}}{W} \neq 0
$$

Under this restriction we may neglect the second and third term between [ ] in (4.70), which simplifies this integral equation considerably. With the complex notations (4.39)-(4.43) and with $J^{(0)} d \lambda=i F^{+} d z$ (see (4.55)) this reduced version of (4.70) can be written in the form of the coupled pair of integral equations 


$$
\begin{aligned}
& \frac{1}{2} f^{(0)}+\operatorname{Re}\left\{\frac{1}{2 \pi i} \int_{C} \frac{1}{z-z_{0}} f^{(0)} d z\right\}=\frac{q}{2} \operatorname{Re}\left\{\frac{1}{\pi i} f \frac{1}{C_{2}} F^{+} d z\right\}, \quad z_{0} \in C_{1}, \\
& \frac{1}{2} f^{(0)}+\operatorname{Re}\left\{\frac{1}{2 \pi i} \int_{C} \frac{1}{z-z_{0}} f^{(0)} d z\right\}=\frac{q}{2} \operatorname{Re}\left\{\frac{1}{\pi i} f \frac{1}{C_{1}} F^{+} d z\right\}, \quad z_{0} \in C_{2} .
\end{aligned}
$$

Equation (4.72) ${ }^{1}$ is, apart from a factor $-q / 2$ in its right-hand side, identical to the relation (4.10.2) for $g_{s}$ in [2]. Moreover, we note that $(4.72)^{2}$ is in accordance with the symmetry relations [2], (4.6) $\left(\mathrm{S}_{\mathrm{e}}, \mathrm{S}_{\mathrm{o}}\right)$, reading

$$
g_{s}(-z)=g_{s}(z),\left(\mathrm{S}_{e}\right) ; \quad g_{s}(-z)=-g_{s}(z),\left(\mathrm{S}_{0}\right) .
$$

Hence, we conclude that

$$
f^{(0)}=-\frac{q}{2} g_{s}
$$

The factor $q / 2$ in (4.73) is due to the fact that we did not a priori put $W_{2}=-W_{1}$, as was done in [2]. The minus-sign is due to the fact that the directions of the current through $\partial D_{1}$, i.e. $\mathbf{e}_{2}$ in [2] and $e_{\phi}$ here, are opposite $\left(e_{2}=-e_{\phi}\right)$.

\subsection{Calculation of the buckling value $I_{0}$}

We start with the calculation of the denominator in the right-hand side of (2.25), which is, apart from a factor $E / 2$, the elastic energy of the pair of tori. From (4.4) we can calculate the components in cylindrical coordinates of the deformations $e_{k l}$ and we find that

$$
\begin{aligned}
& e_{\phi}=-\frac{r-b_{i}}{r b_{i}}\left(w^{\prime \prime}{ }_{i}-v^{\prime}\right)(1+O(\varepsilon)), \\
& e_{r r}=e_{z z}=-v e_{\phi \phi}(1+O(\varepsilon)), \quad e_{r z}=0, \text { in } G_{i}^{-}, i=1,2,
\end{aligned}
$$

while $e_{r \phi}$ and $e_{z \phi}$ are $O(\varepsilon)$ with respect to $e_{\phi \phi}$. With this result the elastic energy becomes

$$
\begin{aligned}
& \frac{1}{1+v} \int_{G_{i}^{-}}\left[\frac{v}{1-2 v} e_{k k} e_{l l}+e_{k l} e_{k l}\right] d V=\int_{D_{i}^{-}} \int_{0}^{2 \pi} \frac{\left(r-b_{i}\right)^{2}}{r^{2} b_{i}{ }^{2}}\left(w^{\prime \prime}{ }_{i}-v_{i}^{\prime}\right)^{2} r d \phi d S(1+O(\varepsilon)), \\
& =\frac{I_{2}}{b^{3}} \int_{0}^{2 \pi}\left(w^{\prime \prime}{ }_{i}+w_{i}\right)^{2} d \phi(1+O(\varepsilon)),
\end{aligned}
$$

where we have used (4.6), the relations $r=b(1+O(\varepsilon)), b_{i}=b(1+O(\varepsilon))$, and the definition

$$
I_{z}:=\int_{D_{i}^{-}}\left(r-b_{i}\right)^{2} d S=\frac{1}{4} \pi a^{4}
$$

We note that (4.76) represents the classical expression for the elastic energy (apart from a factor $E / 2$ ) for in-plane bending of a slender inextensible ring. With $w_{i}$ as given by (4.9) we moreover have

$$
\int_{0}^{2 \pi}\left(w^{\prime \prime}{ }_{i}+w_{i}\right)^{2} d \phi=9 \pi W_{i}^{2} .
$$

For the evaluation of the first term in the first integrand in (2.25) we use (3.1)-(3.2) (in 
dimensionless form, so without the factor $\left.\mu_{0}\right),(4.3),(4.4),(4.9)$ and $(4.10)$ and we neglect $O\left(\varepsilon^{2}\right)$-terms, resulting in

$$
\begin{aligned}
& -\left(\Psi+B_{k} u_{k}\right)_{\perp} B_{l} u_{m} N_{m}=-W \cos 2 \psi\left(f_{,} N_{2}-f_{,} N_{r}\right) U W_{j} \cos 2 \phi N_{r} \\
& =J \frac{d f}{d s} N_{r} W W_{j} \cos ^{2} 2 \phi, \quad \text { on } \partial G_{j}, j=1,2 .
\end{aligned}
$$

Integration over $\partial G_{j}$ of the right-hand side of (4.79) yields (subsequently with use of (4.17), (4.37), (4.69), (4.28), (4.29) and the relation $J_{:}^{(0)} N_{\xi}=-\operatorname{Im} F^{+}$, following from (4.48) and (4.53))

$$
\begin{aligned}
& -\int_{a b_{j}}\left(\Psi+B_{k} u_{k}\right)_{d} B_{l} u_{m} N_{m} d S=\pi W W_{j} \int_{\partial b_{j}} J \frac{d f}{d s} N_{r} r d s= \\
& =\pi b W W_{j} \int_{C_{j}} J^{(0)} \frac{d f(0)}{d \lambda} N_{\xi} d \lambda(1+O(\varepsilon))=-\pi W W_{j} b \operatorname{Im}\left\{\int_{C_{j}} F^{+} \cdot \frac{d f^{(0)}}{d \lambda} d \lambda\right\}, j=1,2,
\end{aligned}
$$

after the omission of $O(\varepsilon)$-terms. For $j=1$, the integral in the right-hand side of (4.80) is computed in [2]; the corresponding result can be obtained from [2], (4.16.2) (with $I_{y}=\pi R / 4$ and $\lambda=\delta / R$ ) and [2], (4.44)-(4.45). Bearing in mind that $f^{(0)}=-98_{s} / 2$, we thus obtain

$$
\operatorname{Im}\left\{\int_{C_{1}} F^{+} \cdot \frac{d f^{(0)}}{d \lambda} d \lambda\right\}= \begin{cases}-\frac{1}{2} q \pi Q, & \left(\mathrm{~S}_{e}\right), \\ \frac{\pi q}{4 \beta^{3}}\left(\alpha+\alpha^{-1}\right), & \left(\mathrm{S}_{\odot}\right),\end{cases}
$$

where

$$
\begin{aligned}
& Q:=\frac{4}{\beta^{2}} \sum_{n=1}^{\infty} \frac{n \alpha^{4 n}\left(1-\alpha^{2 n}\right)^{3}}{\left(1+\alpha^{2 n-2}\right)^{2}\left(1+\alpha^{2 n}\right)^{3}\left(1+\alpha^{2 n+2}\right)^{2}}, \\
& \beta:=\sqrt{m^{2}-1}, \quad \alpha:=m-\beta, \quad m=\frac{c}{a} .
\end{aligned}
$$

The integral for $j=2$ in the right-hand side of (4.80) is the opposite of the one for $j=1$, as follows from the symmetry relations (4.58) and (4.73)-(4.74). Adding the results for $j=1$ and $j=2$ and using $(4.71)$ for $\left(W_{1}-W_{2}\right)$ we finally obtain for the first term in the right-hand side of (2.25)

$$
-\int_{\alpha G}\left(\Psi+B_{k} u_{k}\right)_{m} B_{m} u_{l} N_{l} d S=\left\{\begin{array}{l}
\frac{1}{2} \pi^{2} b q^{2} Q W^{2},\left(S_{)}\right), \\
-\pi^{2} b \frac{q^{2}}{4 \beta^{3}}\left(\alpha+\alpha^{-1}\right) W^{2}, \quad\left(S_{\alpha}\right) .
\end{array}\right.
$$

The calculation of the remaining terms in the right-hand side of (2.25) tums out to be redundant because, as we shall show, these terms are either identically zero or $O\left(\varepsilon^{2}\right)$ with respect to the first term and, hence, they are negligible. The third and fourth term vanish identically; the latter because we have determined $\psi$ exactly. The second term in the integrand is equal to 


$$
\begin{aligned}
& -\frac{1}{2} B_{k} B_{k}\left(u_{l} u_{m}-u_{l}\left(u_{m \downarrow}+u_{l, m}\right)\right) N_{m} \\
& =\frac{1}{2} J^{2} \frac{r-b_{j}}{r b_{j}}\left(w^{\prime \prime}{ }_{j}-v^{\prime}{ }_{j}\right) w_{j} N_{r}, \text { on } \partial G_{j}, j=1,2 .
\end{aligned}
$$

and, hence, the integral over $\partial G$ of this term is $O\left(\varepsilon^{2}\right)$ smaller than the first term, given by the right-hand side of (4.84). Finally, with $T_{r \phi}=T_{\phi z}=0$ and with the use of (4.4) it can be shown that

$$
\int_{G_{j}^{-}} T_{m k} u_{l, m k} u_{l} d V=\frac{1}{2 b} \int_{D_{j}^{-}} T_{\psi} d S \int_{0 .}^{2 \pi} w_{j}\left(w^{\prime \prime}{ }_{j}+w_{j}\right) d \phi\left(1+O\left(\varepsilon^{2}\right)\right), \quad j=1,2 .
$$

Since the nomalized stress $T_{\text {in }}$ is of order unity, the right-hand side of (4.86) has the order of magnitude $a^{2} W^{2} / b$, and, hence, is also $O\left(\varepsilon^{2}\right)$ with respect to the first term. Thus, it is shown that for small $\varepsilon$ the numerator in the right-hand side of $(2.25)$ is indeed dominated by its first term. Substitution of (4.77), (4.78) and (4.84) into (2.25) now yields

$$
\frac{4 \pi^{2} E I_{z} a^{2}}{\mu_{0} J_{0}^{2} b^{4}}= \begin{cases}\frac{1}{18} \pi q^{2} Q, & \left(\mathbf{S}_{e}\right), \\ \frac{1}{36} \frac{\pi}{\beta^{3}} q^{2}\left(\alpha+\alpha^{-1}\right), & \left(\mathbf{S}_{0}\right) .\end{cases}
$$

In the case of equally directed currents the lowest buckling value is found for the highest value of $q^{2}$. According to (4.71) the maximal value of $q^{2}$ is 2 , and occurs for $W_{2}=-W_{1}$, implying that the buckling displacements of the two tori are equal but opposite to each other, in analogy with the results of [2]. This finally results in the following buctling value for $I_{0}$

$$
I_{0}=6 \frac{a}{b^{2}}\left[\frac{\pi E I_{z}}{\mu_{0} Q}\right]^{1 / 2}=\frac{3 \pi a^{3}}{b^{2}}\left[\frac{E}{\mu_{0} Q}\right]^{1 / 2} \text {, (S.). }
$$

In the case of opposite currents the tori do not buckle for $q \neq 0$. If $q=0$, we have to review our analysis, starting from (4.70), in which now the first term between [ ] drops out. The resulting $f^{(0)}$ is now $O\left(\varepsilon^{2}\right)$ smaller and in the computation of the right-hand side of (2.25), the integrals we have neglected before, play a role too. One would then expect a leading term in the righthand side of $(2.25)$ which is $O\left(\varepsilon^{2}\right)$ smaller than in the preceding analysis, but after performing the necessary laborious calculations it appears that this term vanishes too. By means of symmetry relations analogous to (4.58) and (4.73) it is possible to show that the leading term must be $O\left(\varepsilon^{4}\right)$ smaller than in the preceding analysis, which means that if the tori buckle at all, the buckling value is $O\left(\varepsilon^{-2}\right)$ higher than in the case of equally directed currents. 


\section{Two coaxial superconducting tori}

Consider two equal coaxial superconducting tori, which both have a circular cross-section with radius $a$. The central lines of both tori have radius $b$ and the distance between the parallel equatorial planes is $2 c$. A coordinate system $\left\{O \mathbf{e}_{r} \mathbf{e}_{\phi} \mathbf{e}_{z}\right\}$ is chosen with $O$ on the joint axis of the tori midway between the equatorial planes, with $e_{r}$ and $e_{\phi}$ parallel to the equatorial planes and with $e_{z}$ along the joint axis. The corresponding cylindrical coordinates are $(r, \phi, z)$. A cross-section of the pair of tori is shown in Fig. 2.

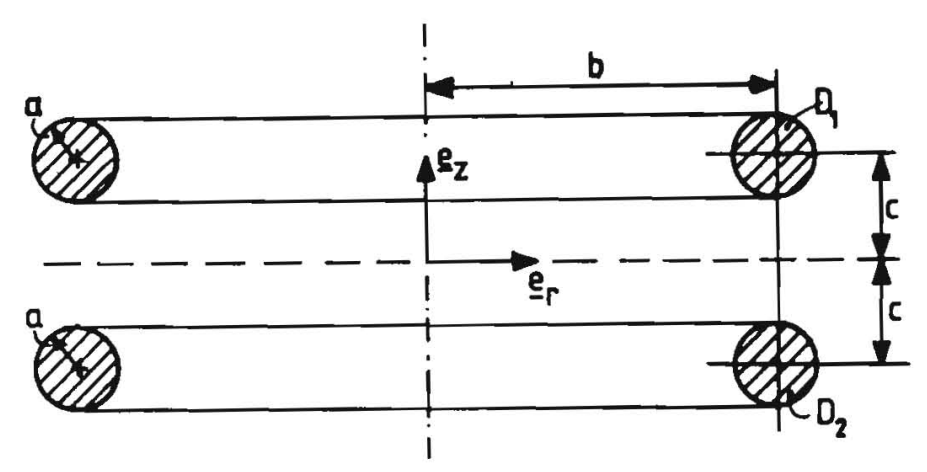

Fig.2. Cross-section of a pair of coaxial tori

Variables pertaining to the upper torus are labelled with an index 1 , and variables pertaining to the lower torus with an index 2. Relations (4.1)-(4.3) remain valid here. The total current on the upper torus is $I_{0}$. The total current on the lower torus is either $I_{0}$ or $-I_{0}$, corresponding to equally directed or oppositely directed currents, respectively.

We assume out-of-plane buckling. The deflection of the central line of either torus is of the form $w_{i}(\phi) e_{z}$ and, moreover, the cross-section rotates about the central line by an angle $\tau_{i}(\phi)$. For a slender ring (i.e. up to $O\left(\varepsilon^{2}\right)$-terms) the displacement field can then be expressed in $w_{i}$ and $\tau_{i}$ as

$$
\begin{aligned}
& u_{r}=\left(z-c_{i}\right) \tau_{i}+v \frac{\left(z-c_{i}\right)(r-b)}{b^{2}}\left(w_{i}^{\prime \prime}-b \tau_{i}\right), \\
& u_{\phi}=-\frac{z-c_{i}}{b} w_{i}^{\prime}, \\
& u_{z}=w_{i}-(r-b) \tau_{i}+\frac{1}{2} v\left[\frac{\left(z-c_{i}\right)^{2}}{b^{2}}-\frac{(r-b)^{2}}{b^{2}}\right]\left(w_{i}^{\prime \prime}-b \tau_{i}\right), \text { in } G_{i}, i=1,2,
\end{aligned}
$$

where

$$
c_{1}=c, c_{2}=-c .
$$

Analogously to (4.9)-(4.10) we find (corresponding to the lowest periodical buckling mode)

$$
\begin{aligned}
& w_{i}(\phi)=W_{i} \cos 2 \phi, \quad \tau_{i}(\phi)=T_{i} \cos 2 \phi, \quad i=1,2, \\
& \tilde{\psi}(r, \phi, z)=f(r, z) W \cos 2 \phi, \quad W:=\sqrt{W_{1}^{2}+W_{2}^{2}} .
\end{aligned}
$$

At the end of this section we establish relationships between the unknowns $W_{1}, W_{2}, T_{1}$ and 
$T_{2}$, again by variation of the right-hand side of (2.25).

As in the preceding section, we construct the integral equations for $J$ and $f$ and linearize them with respect to $\varepsilon$. By means of a simple transformation (a rotation by $1 / 2 \pi$ ) we relate the linearized integral equations to those of the preceding section. The procedure leading to the buckling value is then analogous to the one of the preceding section, except for the calculation of the elastic energy.

\subsection{The zeroeth order approximation of $J(r, z)$}

In subsection 4.1 we did not use the specific form of the contour $C$ until we established the relationship with [2], in the last paragraph of 4.1 (especially in the symmetry relations (4.58)). Therefore, the results of subsection 4.1 can immediately be used here. The only difference lies in the form of the contour $C$, which in Section 4 is defined by (4.42), whereas $C$ is here given by $C_{1} \cup C_{2}$, where $C_{1}$ and $C_{2}$ are the circles

$$
C_{1}:|z-i m|=1, \quad C_{2}:|z+i m|=1 \text {. }
$$

However, by the simple conformal mapping $z \rightarrow \zeta$

$$
\zeta=-i z, \quad z=i \zeta,
$$

the circles $C_{1}$ and $C_{2}$ are mapped onto $\widetilde{C}_{1}$ and $\widetilde{C}_{2}$ respectively, where

$$
\tilde{C}_{1}:|\zeta-m|=1, \quad \tilde{C}_{2}:|\zeta+m|=1 \text {, }
$$

which are identical to $C_{1}$ and $C_{2}$ according to (4.42). The exterior of $\tilde{C}:=\tilde{C}_{1} \cup \tilde{C}_{2}$ in the complex $\zeta$-plane is denoted by $\tilde{S}^{+}$and the interior by $\tilde{S}^{-}$. Furthermore we define

$$
\tilde{F}(\zeta):=i F(i \zeta)
$$

With this definition $\tilde{F}$ satisfies the relations (4.54) and (4.56)-(4.57), with z, $F, C, S$ replaced by $\zeta, \tilde{F}, \tilde{C}, \tilde{S}$. Hence, the function $\tilde{F}$ is identical to the function $F$ used in subsection (4.1) and thus also to the $F$ known from [2].

\subsection{The zeroeth order approximation of $f(r, z)$}

As in 4.2 we take $\phi_{0}=0$, calculate $\left(u\left(x_{0}\right)-u(x)\right) \times J(x)$ and substitute the result together with (4.14)-(4.17) into (3.10), leading to the integral equation (compare with (4.60))

$$
\begin{aligned}
& W f\left(r_{0}, z_{0}\right)=\sum_{j=1}^{2} \frac{1}{2 \pi} \int_{\partial b_{j}} \int_{0}^{2 \pi}\left\{W f(r, z) \cos 2 \phi \frac{N_{r}\left(r_{0} \cos \phi-r\right)+N_{z}\left(z_{0}-z\right)}{R^{3}}\right. \\
& +\left[-\left[\left(W_{i}-\left(r_{0}-b\right) T_{i}\right)-\left(W_{j}-(r-b) T_{j}\right) \cos 2 \phi\right]\left(r_{0} \cos \phi-r\right)\right. \\
& \left.\left.+\left[\left(z_{0}-c_{i}\right) T_{i} \cos \phi-\left(z-c_{j}\right) T_{j} \cos 2 \phi\right]\left(z_{0}-z\right)\right] J\left(r_{,} z\right) \frac{1}{R^{3}}\right\} r d \phi d s,\left(r_{0,} z_{0}\right) \in \partial D_{i} .
\end{aligned}
$$

We carry out the integration over $\phi$ exactly, and with the help of (4.19)-(4.25) and (4.61)(4.64) we arrive at an integral equation which is the analogon of (4.67). Linearization of this equation, again under the supposition that $q$ as defined in (4.71) is unequal to zero and with the introduction of complex coordinates, finally leads to the following coupled pair of integral equations 


$$
\begin{aligned}
& \frac{1}{2} f^{(0)}+\operatorname{Re}\left\{\frac{1}{2 \pi i} \int_{C} \frac{1}{z-z_{0}} f^{(0)} d z\right\}=\frac{q}{2} \operatorname{Re}\left\{\frac{1}{\pi i} f \frac{1}{C_{2}} F^{+} d z\right\}, \quad z_{0} \in C_{1}, \\
& \frac{1}{2} f^{(0)}+\operatorname{Re}\left\{\frac{1}{2 \pi i} \int_{C} \frac{1}{z-z_{0}} f^{(0)} d z\right\}=-\frac{q}{2} \operatorname{Re}\left\{\frac{1}{\pi i} f \frac{1}{C_{1}} F^{+} d z\right\}, \quad z_{0} \in C_{2} .
\end{aligned}
$$

Introducing $\zeta$ by $(5.6), \tilde{F}(\zeta)$ by $(5.8)$ and $\tilde{f}^{(0)}(\zeta)$ by

$$
\tilde{f}^{(0)}(\zeta):=f^{(0)}(i \zeta)
$$

we can confirm that $f^{(0)}$ satisfies the relations (4.70), with $z, F, f^{(0)}, C$ replaced by $\zeta, \tilde{F}, \tilde{f}^{(0)}, \tilde{C}$. Hence, the function $\tilde{f}^{(0)}$ is identical to the function $f^{(0)}$ from subsection 4.2 , and thus also related to the function $g_{s}$, calculated in [2].

\subsection{Calculation of the buckling value $I_{0}$}

The displacement field (5.1) yields the following, well-known, expression for the elastic energy for a slender ring in out-of-plane bending

$$
\begin{aligned}
& \frac{1}{1+v} \int_{G_{i}^{-}}\left[\frac{v}{1-2 v} e_{k k} e_{l l}+e_{k l} e_{k l}\right] d V \\
& =\frac{I_{r}}{b^{3}} \int_{0}^{2 \pi}\left(w^{\prime \prime}{ }_{i}-\tau_{i} b\right)^{2} d \phi+\frac{I_{p}}{2(1+v) b^{3}} \int_{0}^{2 \pi}\left(w_{i}^{\prime}+\tau_{i} b\right)^{2} d \phi \\
& =\frac{\pi I_{r}}{b^{3}}\left(4 W_{i}+T_{i} b\right)^{2}+\frac{\pi I_{p}}{2(1+v) b^{3}}\left(2 W_{i}+2 T_{i} b\right)^{2}, \quad i=1,2,
\end{aligned}
$$

where in the last step (5.3) is used, and where

$$
\begin{aligned}
& I_{r}:=\int_{D_{i}^{-}}\left(z-c_{i}\right)^{2} d S=\frac{1}{4} \pi a^{4}, \\
& I_{p}:=\int_{D_{i}^{-}}\left((r-b)^{2}+\left(z-c_{i}\right)^{2}\right) d S=\frac{1}{2} \pi a^{4} .
\end{aligned}
$$

For the first term in the first integrand in (2.25) we find analogously to (4.79)

$$
\begin{aligned}
& -\left(\psi+B_{k} u_{k}\right)_{l} B_{l} u_{m} N_{m}=J \frac{d f}{d s} W \cos ^{2} 2 \phi\left[\left(z-c_{j}\right) T_{j} N_{r}+\left(W_{j}-(r-b) T_{j}\right) N_{z}\right]= \\
& =J^{(0)} \frac{d f^{(0)}}{d s} W \cos ^{2} 2 \phi W_{j} N_{z}(1+O(\varepsilon)), \quad \text { on } \partial G_{j}, j=1,2,
\end{aligned}
$$

as we assume that $T_{j}=O(W / b)$ as is suggested by the specific form of the result (5.12), and as will be confirmed furtheron (see (5.18)). Integration over $\partial G$ of the right-hand side of (5.14) yields (analogously to (4.80), but now with the use of $J^{(0)} N_{\eta}=-\operatorname{Im}\left(i F^{+}\right)$)

$$
-\int_{a b_{j}}\left(\Psi+B_{k} u_{k}\right)_{\downarrow} B_{l} u_{m} N_{m} d S=\pi W W_{j} b \int_{C_{j}} J^{(0)} \frac{d f^{(0)}}{d \lambda} N_{\eta} d \lambda=
$$




$$
=-\pi W W_{j} b \operatorname{Im}\left\{i \int_{C_{j}} F^{+} \frac{d f^{(0)}}{d \lambda} d \lambda\right\}=-\pi W W_{j} b \operatorname{Im}\left\{\int_{\bar{C}_{j}} \tilde{F}^{+} \frac{d \tilde{f}^{(0)}}{d \lambda} d \lambda\right\},
$$

with $\tilde{C}_{j}, \tilde{F}^{+}$and $\tilde{f}^{(0)}$ according to (5.7), (5.8) and (5.11). The conclusions at the end of subsections 5.1 and 5.2 imply that the right-hand side of (5.15) is exactly equal to the right-hand side of (4.80) and, consequently, the result (4.84) for the integral over the first term in the right-hand side of (2.25) holds here, too. As in subsection 4.3 it can be shown that all other terms in the numerator of (2.25) may be neglected.

Using $I_{p}=2 I_{r}$ in (5.12) and then substituting (5.12) and (4.84) into (2.25), we obtain

$$
\frac{4 \pi^{2} E I_{r} a^{2}}{\mu_{0} I_{0}^{2} b^{4}}= \begin{cases}\frac{\pi}{2} q^{2} Q p^{-1}, \quad\left(\mathbf{S}_{\mathrm{e}}\right), \\ \frac{\pi}{4 \beta^{3}} q^{2}\left(\alpha+\alpha^{-1}\right) p^{-1}, & \left(\mathbf{S}_{\mathrm{o}}\right),\end{cases}
$$

where

$$
p=W^{-2} \sum_{i=1}^{2}\left[\left(4 W_{i}+T_{i} b\right)^{2}+\frac{1}{1+v}\left(2 W_{i}+2 T_{i} b\right)^{2}\right] .
$$

In the case of equally directed currents, minimization of $p$ with respect to $T_{1}$ and $T_{2}$ yields

$$
T_{i} b=\frac{4(2+v)}{5+v} W_{i}, i=1,2 \text {, and } p=\frac{36}{5+v} .
$$

Since $q^{2} \leq 2$ (see (4.71)), the right-hand side of (5.16) $\left(\mathbf{S}_{e}\right)$ attains its maximum value for $q^{2}=2$, corresponding to $W_{2}=-W_{1}$. Hence, the buckling displacements are again opposite to each other. The lowest buckling value is thus found to be

$$
I_{0}=\frac{12}{\sqrt{5+v}} \frac{a}{b^{2}}\left[\frac{\pi E I_{r}}{\mu_{0} Q}\right]^{3 / 2}=\frac{6 \pi a^{3}}{\sqrt{5+v} b^{2}}\left[\frac{E}{\mu_{0} Q}\right]^{1 / 2}, \quad\left(\mathbf{S}_{\mathrm{e}}\right) .
$$

In the case of opposite currents, the tori do not buckle for $q \neq 0$. If $q=0$, then we have to review the calculation of $f^{(0)}$. The resulting buckling value will again be much higher than for equally directed currents. Since the calculations involved are massive, and the results of little practical use, we refrain from dilating upon this calculation. 


\section{Conclusions and discussion}

In the preceding two sections we have calculated the buckling current for sets of two concentric and two coaxial tori. In both cases the electric currents through the tori are equal, both in magnitude and in direction. The results, which are given by the formulae (4.88) and (5.19), are recapitulated below:

$$
I_{0}=\frac{3 \pi a^{3}}{b^{2}}\left[\frac{E}{\mu_{0} Q}\right]^{1 / 2},
$$

for a pair of concentric tori, and

$$
I_{0}=\frac{6 \pi a^{3}}{\sqrt{5+v b^{2}}}\left[\frac{E}{\mu_{0} Q}\right]^{1 / 2} \text {, }
$$

for a pair of coaxial tori. In [2], a table showing values of $Q$ as function of $m=c / a$ is given (cf. [2], Table 4). The above results are visualized in Figure 3. Here we have used the following numerical values

$$
\begin{aligned}
& E=8 \times 10^{10} \mathrm{~N} / \mathrm{m}^{2} ; \quad \mu_{0}=4 \pi \times 10^{-7} \mathrm{H} / \mathrm{m} ; \quad \mathrm{v}=0.3 ; \\
& b=0.5 \mathrm{~m} ; \quad a=5 \times 10^{-3} \mathrm{~m} .
\end{aligned}
$$

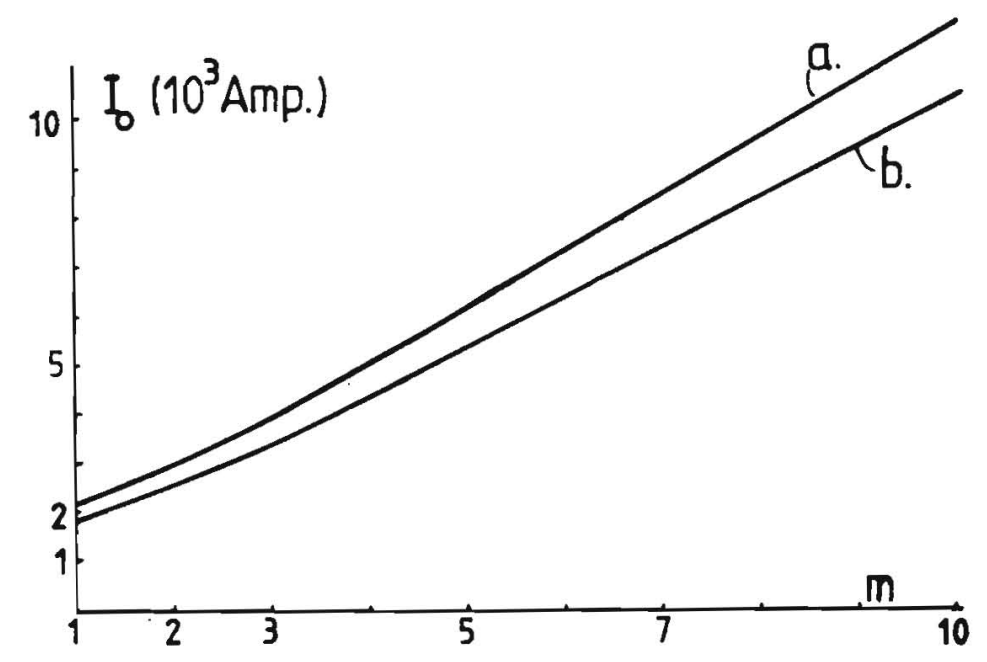

Fig.3. Buckling current as function of $m$ ( $a$ : concentric pair; $b$ : coaxial pair)

In [2] the buckling current for a pair of parallel straight beams was calculated. The final result, according to $[2],(5.17)$, reads

$$
I_{0}=\frac{\pi^{3} R^{3}}{l^{2}}\left[\frac{E}{\mu_{0} Q}\right]^{1 / 2} \text {. }
$$

One can compare the result (6.3) with (6.1) and (6.2) by realizing that $R$ and $l$ must be related to $a$ and $b$. By taking $a=R$ and $\pi b=2 l$ (leading to equal periods for the buckling modes for the beam and the torus) we find that the buckling values according to (6.1) and (6.2) are a factor $3 / 4$ and $3 / 2 \sqrt{5+v}$, respectively, times the buckling value (6.3). Hence, we notice that the buckling values for pairs of parallel beams, concentric tori or coaxial tori all differ only a numerical factor from each other. Moreover, these numerical factors are completely determined by the elastic energies of the respective systems (see (4.76), (5.12) and [2], (2.2)). This is due to the fact that the term which in fact is determinant for the buckling value, i.e. the numerator of the right-hand side of (2.25), for slender pairs of beams is dominated by its first term. This term takes the same value for all of the three systems mentioned above, at least in a zeroeth 
order approximation with respect to $\varepsilon$ (see the comments in the final paragraphs of the Subsections 4.1, 4.2, 5.1 and 5.2). Therefore, it is expected that the buckling value for any "slender" pair of parallel curved beams is equal to that of an equivalent pair of straight beams times the ratio of the elastic energies. The concept "slenderness" has to be defined properly in each problem in hand.

In [2], a more simple, but less accurate, method for the solution of our buckling problem was presented. The method is based upon a generalization of the law of Biot and Savart, as described by Moon, [3], Sect. 2.6. It was shown in [2] how this method yields approximate buckling values $I_{0}$ for a set of two parallel rods, which are very close to the exact values as long as the two rods are not too near. These results were derived from the basic relation, [2], (5.18), for the force on one current-carrying curve $L_{1}$ due to the current in a second curve $L_{2}$.

Let us now apply this relation to the buckling problems described in the Sections 4 and 5 of the present paper. We start with the system of Section 4 as illustrated in Fig.1. The tori are considered as one-dimensional circles (rings) $L_{1}$ and $L_{2}$, which can be described by the sets of cylindrical coordinates $\left\{r_{1}, \phi_{1}, z_{1}\right\}$ and $\left\{r_{2}, \phi_{2}, z_{2}\right\}$, with bases $\left\{\mathbf{e}_{r_{1}}, \mathbf{e}_{\phi_{1}}, \mathbf{e}_{z}\right\}$ and $\left\{\mathbf{e}_{r_{2}}, \mathbf{e}_{\phi_{2}}, \mathbf{e}_{2}\right\}$, respectively. In the undeformed state of $L_{1}$ and $L_{2}$ one has $r_{i}=b_{i}, z_{i}=0$ and $\phi_{i} \in[0,2 \pi], i=1,2$. Restricting ourselves to in-plane bending, with the displacements of the central lines of the tori according to (4.4), we find for the position vectors $r_{1}$ and $r_{2}$ of two points $P_{1} \in L_{1}$ and $P_{2} \in L_{2}$, respectively, the relations

$$
\begin{aligned}
& \mathbf{r}_{1}=\left(b_{1}+w_{1}\left(\phi_{1}\right)\right) \mathbf{e}_{r_{1}}+v_{1}\left(\phi_{1}\right) \mathbf{e}_{\phi_{1}}, \\
& \mathbf{r}_{2}=\left(b_{2}+w_{2}\left(\phi_{2}\right)\right) \mathbf{e}_{r_{2}}+v_{2}\left(\phi_{2}\right) \mathbf{e}_{\phi_{2}} .
\end{aligned}
$$

The unit tangent vectors $t_{1}$ and $t_{2}$ along $L_{1}$ and $L_{2}$, respectively, and the position vector $\mathbf{R}$ from $P_{2}$ to $P_{1}$ are given by [2], (5.19), and they become here (the inextensibility condition (4.6) is already taken into account)

$$
\begin{aligned}
& \mathbf{t}_{1}=\mathbf{e}_{\phi_{1}}+\frac{1}{b_{1}}\left[w_{1}^{\prime}\left(\phi_{1}\right)-v_{1}\left(\phi_{1}\right)\right] \mathbf{e}_{r_{1}}, \\
& \mathbf{t}_{2}=\mathbf{e}_{\phi_{2}}+\frac{1}{b_{2}}\left[w_{2}^{\prime}\left(\phi_{2}\right)-v_{2}\left(\phi_{2}\right)\right] \mathbf{e}_{r_{2}}, \\
& \mathbf{R}=\left[b_{1}+w_{1}\left(\phi_{1}\right)\right] \mathbf{e}_{r_{1}}-\left[b_{2}+w_{2}\left(\phi_{2}\right)\right] \mathbf{e}_{r_{2}}+v_{1}\left(\phi_{1}\right) \mathbf{e}_{\phi_{1}}-v_{2}\left(\phi_{2}\right) \mathbf{e}_{\phi_{2}} .
\end{aligned}
$$

These relations must be substituted into the force-relation [2], (5.18), and then the result must be linearized with respect to the small displacements $u_{i}$ and $v_{i}$. This ultimately results in an expression for the force on the ring $L_{1}$ of the form [2], (5.23), of which only the linear contribution $f$ is relevant. The calculation of this term is somewhat cumbersome but straightforward, and therefore we omit the underlying calculations. We only have to mention that in these calculations it has been assumed that $c / b \ll 1$ (being the criterion for the slendemess of the pair of rings), and that we have neglected all terms that are $o(1)$ for $c / b \rightarrow 0$. This finally results in the following expression for the force per unit of length acting in $P_{1}$ on $L_{1}$,

$$
\mathbf{f}\left(\phi_{1}\right)=f\left(\phi_{1}\right) \mathbf{e}_{r_{1}}=\frac{\mu_{0} I_{0}^{2}}{8 \pi c^{2}}\left[w_{1}\left(\phi_{1}\right)-w_{2}\left(\phi_{1}\right)\right] \mathbf{e}_{r_{1}} .
$$

This purely radial load serves as the load parameter in the ring equation, which for an inextensible ring in in-plane bending reads (cf. [3], Sect. 6.7, or [5], (7.2)) 


$$
\begin{aligned}
& w_{1}^{\mathrm{v}}\left(\phi_{1}\right)+2 w_{1}^{\prime \prime \prime}\left(\phi_{1}\right)+w_{1}^{\prime}\left(\phi_{1}\right)=\frac{b^{4}}{E I} f^{\prime}\left(\phi_{1}\right) \\
& =\frac{\mu_{0} I_{0}^{2}}{\pi^{2} E}\left[\frac{b^{2}}{c a^{2}}\right]^{2} \frac{1}{2}\left[w_{1}^{\prime}\left(\phi_{1}\right)-w_{2}^{\prime}\left(\phi_{1}\right)\right] .
\end{aligned}
$$

An analogous ring equation holds for $w_{2}\left(\phi_{2}\right)$ on $L_{2}$. The lowest buckling value (for a periodical buckling mode) corresponds to

$$
w_{1}(\phi)=-w_{2}(\phi)=W \cos 2 \phi,
$$

(in accordance with $q^{2}=2$ and (4.9)) and this yields

$$
I_{0}=\frac{3 \pi a^{2} c}{b^{2}}\left[\frac{E}{\mu_{0}}\right]^{1 / 2} \text {. }
$$

This result is in agreement with (4.88) if in the latter $1 / \sqrt{Q}$ is replaced by $c / a$. As already shown in [2], at the end of Section 5, this is approximately true for c/a not too close to unity (e.g. for $c / a \geq 4$ the relative difference is less than $5 \%$ ). The worst discrepancy occurs for $c / a \rightarrow 1$; in that case relation (6.9) gives a buckling value that is about $45 \%$ lower than the one according to (4.88), or, equivalently, (4.88) is $80 \%$ higher than (6.9).

The above method can also be applied to the buckling problem of Section 5 . For this system (see Fig.2) and for out-of-plane buckling (see (5.1)) one has

$$
\begin{aligned}
& \mathbf{r}_{1}=b \mathbf{e}_{r_{1}}+\left[c+w_{1}\left(\phi_{1}\right)\right] \mathbf{e}_{2}, \\
& \mathbf{r}_{2}=b \mathbf{e}_{r_{2}}+\left[-c+w_{2}\left(\phi_{2}\right)\right] \mathbf{e}_{2} .
\end{aligned}
$$

In exactly the same way as in the preceding problem an expression for the linearized perturbed force can be derived. In this case the force is in the $\mathbf{e}_{2}$-direction and is equal to (under the neglect of $O(c / b)$-terms)

$$
f_{2}\left(\phi_{1}\right)=\frac{\mu_{0} I_{0}^{2}}{8 \pi c^{2}}\left[w_{1}\left(\phi_{1}\right)-w_{2}\left(\phi_{1}\right)\right] .
$$

The ring equations for out-of-plane bending and torsion can be found in [3], (6-7.18). With the substitutions

$$
\begin{aligned}
& A=E I, \quad C=G I_{p}\left[=\frac{E I}{1+v}\right], \\
& u=-w_{1}\left(\phi_{1}\right), \quad \phi=-\tau_{1}\left(\phi_{1}\right), \quad '=\frac{d}{d z}=\frac{1}{b} \frac{d}{d \phi_{1}},
\end{aligned}
$$

these relations become

$$
\begin{aligned}
& -\frac{E I}{b^{4}}\left[w_{1}^{\mathrm{IV}}\left(\phi_{1}\right)-b \tau_{1}^{\prime \prime}\left(\phi_{1}\right)\right]+\frac{G I_{p}}{b^{4}}\left[w_{1}^{\prime \prime}\left(\phi_{1}\right)+b \tau_{1}^{\prime \prime}\left(\phi_{1}\right)\right]+f_{z}\left(\phi_{1}\right)=0, \\
& -\frac{E I}{b^{2}}\left[w_{1}^{\prime \prime}\left(\phi_{1}\right)-b \tau_{1}\left(\phi_{1}\right)\right]-\frac{G I_{p}}{b^{2}}\left[w_{1}^{\prime \prime}\left(\phi_{1}\right)+b \tau_{1}^{\prime \prime}\left(\phi_{1}\right)\right]=0 .
\end{aligned}
$$

Using 


$$
G I_{p}=\frac{E I}{1+v},
$$

and the relations (5.3) for $w_{1}$ and $\tau_{1}$, we obtain from the second relation of (6.13) (in accordance with $(5.18)^{2}$ )

$$
T_{1}=-\frac{4(2+v)}{5+v} \frac{W_{1}}{b} .
$$

With this result the first relation of (6.13) yields

$$
\frac{36 E I}{(5+v) b^{4}} W_{1} \cos 2 \phi=f_{z}(\phi)=\frac{\mu_{0} l_{0}^{2}}{4 \pi c^{2}} \frac{1}{2}\left(W_{1}-W_{2}\right) \cos 2 \phi \text {. }
$$

An analogous relation holds for $W_{2}$, and it is then easily seen that the lowest buckling value occurs for $W_{2}=-W_{1}$ and is equal to (with $I=\pi a^{4} / 4$ )

$$
I_{0}=\frac{6 \pi a^{2} c}{\sqrt{5+v} b^{2}}\left[\frac{E}{\mu_{0}}\right]^{1 / 2} \text {. }
$$

Again, this result is in agreement with (5.19) if $1 / \sqrt{Q}=c / a$. 


\section{References}

1. P.H. van Lieshout, P.M.J. Rongen and A.A.F. van de Ven, A variational principle for magnetoelastic buckling, J. Eng. Math. 3 (1987) 227-252.

2. P.H. van Lieshout, P.M.J. Rongen and A.A.F. van de Ven, A variational approach to magnetoelastic interaction problems for a system of ferromagnetic or superconducting beams, J. Eng. Math. 4 (1988) ...

3. F.C. Moon, Magneto-solid mechanics, Wiley, New York, 1984.

4. S. Chattopadhyay, Magnetoelastic instability of structures carrying electric current, Int. J. Solid. Struct. 15 (1979) 467-477.

5. A.A.F. van de Ven and M.J.H. Couwenberg, Magnetoelastic stability of a superconducting ring in its own field, J. Eng. Math. 20 (1986) 251-270.

6. R. Courant and D. Hilbert, Methods of mathematical physics, volume I, Interscience publishers, inc., New York, 1953.

7. J.A. Stratton, Electromagnetic theory, International series in pure and applied physics, McGraw-Hill, New York, 1941.

8. S.G. Mikhlin, An advanced course of mathematical physics, North-Holland Publishing Company, Amsterdam, London, 1970.

9. I.S. Gradshteyn and I.M. Ryzhik, Table of integrals, series, and products, Corrected and enlarged edition, Academic Press, London, 1980.

10. N.I. Muskhelishvili, Singular integral equations, Second edition, Noordhoff, Groningen, 1953. 